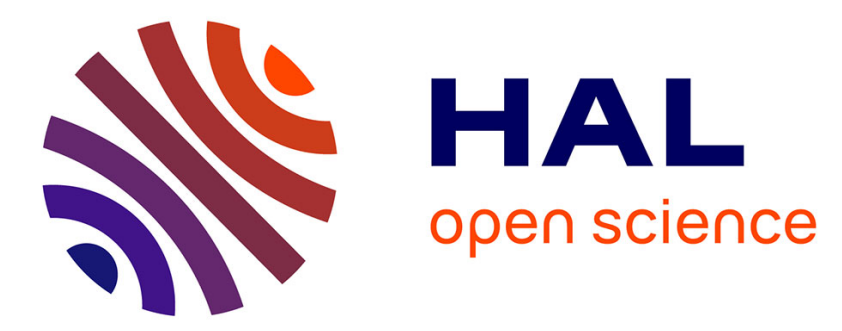

\title{
Aqueduc et structures hydrauliques de la villa de Richeaume I à Puyloubier (Bouches-du-Rhône)
}

Florence Mocci, Kevin Walsh, Vincent Dumas, Jean-Marie Gassend, Cécile Allinne, Alain Badie, Cécile Miramont, Jean-Louis Paillet, Cécile André

\section{- To cite this version:}

Florence Mocci, Kevin Walsh, Vincent Dumas, Jean-Marie Gassend, Cécile Allinne, et al.. Aqueduc et structures hydrauliques de la villa de Richeaume I à Puyloubier (Bouches-du-Rhône). Gallia - Archéologie de la France antique, 2005, Aqueducs de la Gaule méditerranéenne, 62, pp.147-160. 10.3406/galia.2005.3227 . hal-00277823

\section{HAL Id: hal-00277823 \\ https://hal.science/hal-00277823}

Submitted on 9 Jan 2020

HAL is a multi-disciplinary open access archive for the deposit and dissemination of scientific research documents, whether they are published or not. The documents may come from teaching and research institutions in France or abroad, or from public or private research centers.
L'archive ouverte pluridisciplinaire HAL, est destinée au dépôt et à la diffusion de documents scientifiques de niveau recherche, publiés ou non, émanant des établissements d'enseignement et de recherche français ou étrangers, des laboratoires publics ou privés.

\section{(ㅇ)(1) $\$$}

Distributed under a Creative Commons Attribution - NonCommercial - NoDerivatives| 4.0 


\title{
AQUEDUCS ET STRUCTURES HYDRAULIQUES DE LA VILLA DE RICHEAUME I À PUYLOUBIER (BOUCHES-DU-RHÔNE)
}

\author{
Florence MOCCI, Kevin WALSH, Vincent DUMAS et Jean-Marie GASSEND \\ avec la collaboration de Cécile ALlinNE, Alain BADIE, Cécile MiRAMONT, \\ Jean-Louis PAILLET et Cécile ANDRÉ
}

\begin{abstract}
Mots-clés. Aqueduc, structures hydrauliques, villa, Haut-Empire, Antiquité tardive, paléochenal, source.
Résumé. Une fouille archéologique programmée, entreprise de 1998 à 2003 sur le site antique de Richeaume I (Puyloubier, Bouches-duRhône), a révélé la présence d'une vaste villa de plus de $5000 \mathrm{~m}^{2}$ occupée du $\mathrm{I}^{p r}$ s. av. J.-C. au début du VT s. apr: J.-C., alimentée par un aqueduc captant les eaux de source au nord et entourée d'un certain nombre d'élablissements ruraux souvent hérités du second âge du Fèr: Les vestiges archéologiques mis au jour sur une superficie de $2000 \mathrm{~m}^{2}$ correspondent aux parties résidentielle et agricole de la villa du Haut-Empire el de l'Antiquité tardive ainsi qu'à divers aménagements hydrauliques : bassins d'agrément, canalisations, égouts, murs de digue, drains et vestiges d'aqueducs. L'étude proposée ici porte sur les structures hydrauliques découvertes par la prospection géophysique. et les fouilles archéologiques sur le versant oriental du site entre 315,94 et 314,05 m d'altitude. Parmi ces structures (mur de digue, paléochenal, tronsons d'aqueduc) se distinguent plus particulièrement les vestiges d'un aqueduc qui ne peut être mis en relation avec l'alimentation en eau des parties résidentielle et agricole de la villa situées plus en amont. Cet ouvrage, dont la pente à forte déclivité a nécessité la construction d'une imposante substruction, pourrait être associé à l'alimentation en eau d'un des bâtiments voisins dont la nature reste à définir:
\end{abstract}

Key-words. Aqueduct, hydrological structures, villa, High Empire, Late Antiquity, palaeochannel, spring.

Abstract. A research excavation on the Richeaume I site (Puyloubier, Bouches-du-Rhône) took place between 1998 and 2003. This revealed the presence of a substantial villa, covering an area of $5000 \mathrm{~m}^{2}$ that was occupied from the $1^{\text {rst }}$ century $B C$ through to the $4^{\text {th }}$ century $A D$. An aqueduct, which channelled water from a number of springs to the north of the site, supplied the site. A number of other sites that were initially settled during the late Iron Age surround Richeaume I. The archaeological remains excavated covered an area of $2000 \mathrm{~m}^{2}$ and revealed elements of the agricultural and domestic areas of the villa, as well as hydrological structures (ornamental pond, water conduits, sewers, dykes, drains and aqueduct remains). This article presents an analysis of the hydrological structures discovered by geophysical survey, which were subsequently excavated, on the western side of the site between 315,94 $\mathrm{m}$ and 314,05 $\mathrm{m}$ above sea level. Within these structures (dyke, palaeochannel and sections of aqueduct) the most important element is an aqueduct that cannot be directly associated with the supply of water to the residential and agricultural parts of the villa upstream. The presence of this structure, which is characterised by a substantial slope, required the construction of a substantial foundation wall. Therefore, this structure could have been associated with the water supply to one of the adjacent buildings whose characteristics still need to be ascertained.

Translation : Kevin WAL.SH

Schlagwörter. Römische Wasserleitung, wasserbauliche Anlagen, Villa, frühe Kaiserzeit, Spätantike, Gewässeraltarm, Quelle, Puyloubier.

Zusammenfassung. Eine von 1998 bis 2003 an der antiken Fundstelle von Richeaume I (Puyloubier, Dép. Bouches-du-Rhône) durchgeführte Plangrabung führte zur Entdeckung einer ausgedehnten Villenanlage. Sie nimmt eine Fläche von mehr als $5.000 \mathrm{~m}^{2}$ ein und war vom ersten Jahrhundert v. Chr. bis zu Beginn des sechsten Jahrhunderts $n$. Chr. belegt. Sie war durch eine Wasserleitung versorgt, die die Quellen im Norden des Geländes faßl, und von einer bestimmten Anzahl ländlicher Siedelplätze umgeben, die häufig schon in der jüngeren Fisenzeit bestanden. Die auf $2.000 \mathrm{~m}^{2}$ freigelegten archäologischen Überreste betreffen die Wohnbereiche und die landwirtschaftlichen Bereiche der frühkaiserzeitlichen und spätantiken Villa sowie verschiedene wasserbauliche Vorrichtungen (Zierbecken, Leitungskanäle, Abwasserleitungen, Staumauern, Entwässerungsgräben und Reste der Wasserleitung). Die hier vorgetragenen Überlegungen beziehen sich auf die wasserbaulichen Anlagen, die durch geophysikalische Prospektion und archäologische Grabungen auf 
dem Osthang des Platzes zwischen 315,94 m und 314,05 m ü. N. N. entdeckt wurden. Von diesen Anlagen (Staumauer, Altarm eines Gewässerlaufs, Abschnitte der Wasserleitung) fallen insbesondere Überreste einer Wasserleitung auf, die man nur mit der Wasserversorgung der Wohn- und Stallbereiche der weiter bergauf gelegenen Villa in Verbindung bringen kann. Die beschriebene Vorrichtung, bei der die stark abschüssige Hanglage die Errichtung eines gewaltigen Unterbaus erzwang, dürfte mit der Versorgung eines der benachbarten Gebäude zusammenhängen, dessen genaue Funktion noch zu untersuchen wäre.

Übersetzung : Stefan WIRTH

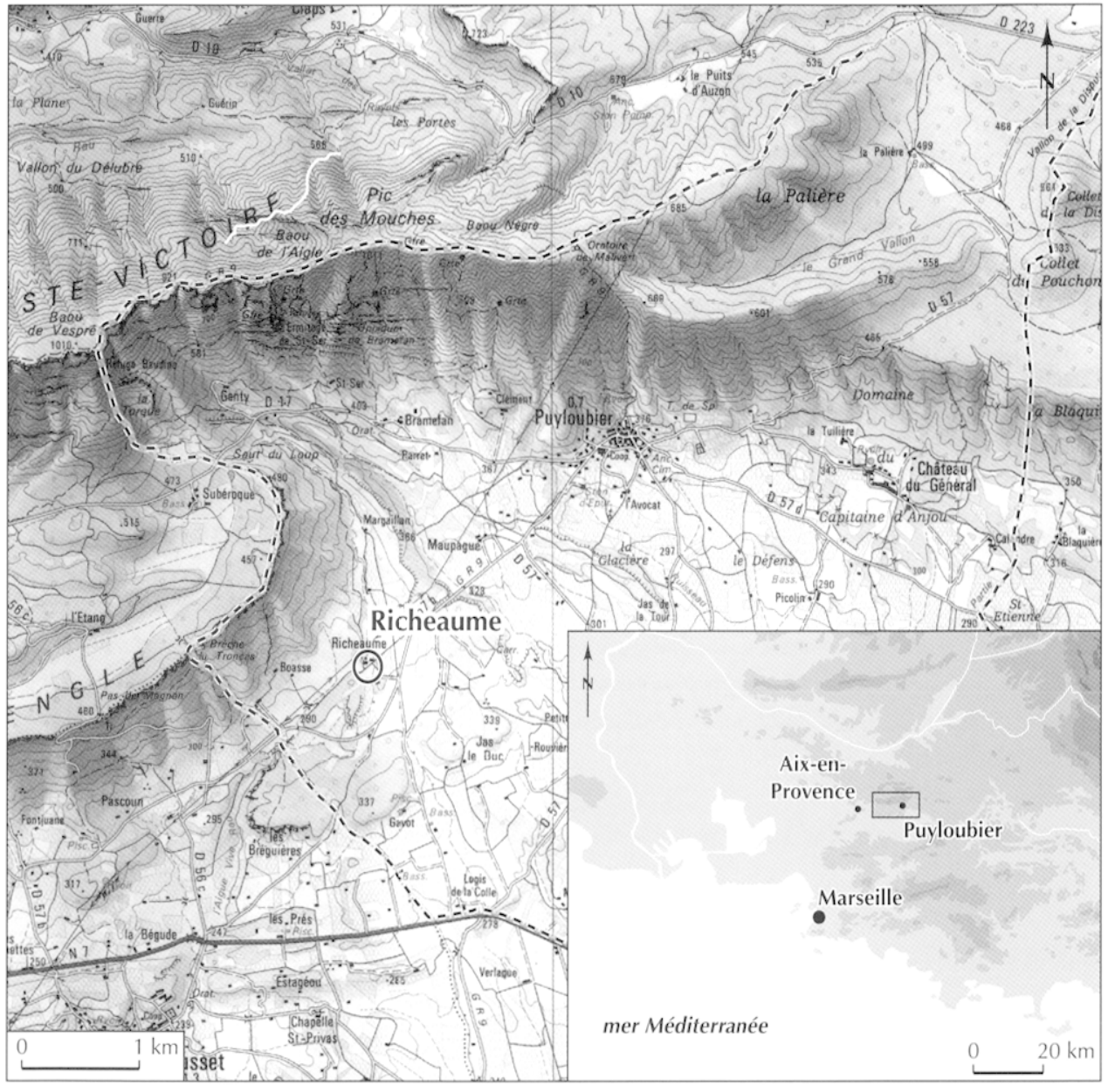

Fig. 134 - Localisation de la commune de Puyloubier et du site. de Richeaume I dans les Bouchesdu-Rhône (extrait de la carte ICN au 1/50 000: Aix-en-Provence; DAO V. Dumas, CNRS).
Depuis 1998, la villa de Richeaume I fait l'objet d'une fouille archéologique programméc accompagnée de sondages géoarchéologiques et de prospections géophysiques ${ }^{108}$, sous la direction de F. Mocci. Située à l'extrémité orientale du bassin d'Aix-en-Provence, dans la haute vallée de l'Arc, cette villa est localisée sur le domaine du même nom, sur le piémont méridional du massif de la Sainte-Victoire et du plateau du Cengle, à l'ouest de la commune de Puvloubier (fig. 134). Ce site est implanté dans le bassin versant du ruisseau de la Naïsse, sur une éminence orientée nord-sud (altitude moyemne: $319 \mathrm{~m}$ ),

108. Ces sondages ont été réalisés avec la collaboration de C.. Allinne (université de Provence), C. Miramont (université de Provence) et K. Walsh (université de York) ; les prospections géophysiques ont été menées en 1999 et 2000 par I. Aubry (societé Terra Nova, Paris) (Mocci, 2000, p. 23 et annexe II). correspondant à la terminaison périclinale du synclinal du Cengle constituée d'argiles, de calcaires et de grès. I'habitat antique se situe ainsi sur la marge des épandages limonocaillouteux pléistocènes dans le souci de contrôler, mais aussi de préserver, les terroirs les plus favorables à la mise en culture (Jorda, Mocci, 1997, p. 224-227). Au nord du site (cotes: $336-370 \mathrm{~m} \mathrm{~N}(\mathrm{~F}$ ) , des sources captées sur le contrefort oriental du Cengle alimentent encore la bastide ${ }^{109}$ et ses dépendances élevées en grande partie sur les constructions antiques, depuis la période médiévale.

109. Cette canalisation contemporaine, de plus de $700 \mathrm{~m}$ de long, a été éleve sur une substruction moderne qui pourrait reprendre le trace d'un aqueduc antérieur (médiéval et/ou antique ?े). I a proposition de restiution du tracé de l'aqueduc principal de la villa s'appuie en partic sur cette hypothese (fig. 135). 
La première mention de "vestiges de constructions antiques et d'aqueducs visibles au sud de la ferme " remonte au XIX ${ }^{\mathrm{e}} \mathrm{s}$. (Chaillan, 1899, p. 87 ; Gilles, 1904, p. 105 ; Benoit, Blanchet, 1936, p. 54). À l'intérieur de cette ferme, lors de travaux de rénovation dans l'étable et la bergerie entrepris en 1972 par M. Hoesch, propriétaire actuel du domaine, un puits médiéval (P1) et quatre pièces ont été mis au jour; une de ces pièces contenait des pilettes d'hypocaustes probablement en place.

L'ensemble des données acquises depuis 1998 révèle la présence d'une vaste villa de plus $5000 \mathrm{~m}^{2}$ occupée du $\mathrm{I}^{\mathrm{er}} \mathrm{s}$. av. J.-C. au début du $v^{\mathrm{e}}$ s. apr. J.-C., alimentée par un aqueduc captant les eaux de source au nord et entourée de plusieurs établissements ruraux souvent hérités du second âge du Fer (fig. 135). Les vestiges archéologiques mis au jour se répartissent sur une superficie de $2000 \mathrm{~m}^{2}$, au sud et à l'est de la bastide actuelle, autour d'une aire de battage médiévale et moderne de $900 \mathrm{~m}^{2}$ (cote : 318,85 m NGF) (fig. 136 et 137). Ces vestiges correspondent aux parties résidentielle et agricole de la villa du Haut-Empire et de l'Antiquité tardive (zones I, II et IV) ainsi qu'à divers aménagements hydrauliques : bassins d'agrément (Bl et B2), canalisations, égouts (zones I, II et IV), murs de digue, drains et vestiges d'aqueducs (zones III et IV).

L'étude proposée dans ce dossier porte sur les structures hydrauliques découvertes par la prospection géophysique et les fouilles archéologiques, sur le versant oriental du site entre $315,94 \mathrm{~m}$ et $314,05 \mathrm{~m}$ d'altitude (zone III) ${ }^{110}$. La zone III (sondages 10,12, 13, 22 et 22a) est limitée à l'ouest par un mur de terrasse contemporain, au sud par une parcelle de vigne, à l'est par le ruisseau de la Naïsse et au nord par un bassin moderne et une aire de stationnement. Au sein de ces structures hydrauliques (mur de digue, paléochenal, tronçons d'aqueducs $\mathrm{C} 4 \mathrm{a}, \mathrm{C} 4 \mathrm{~b}$ et $\mathrm{C} 6$ ) se distinguent plus particulièrement les substructions d'un aqueduc (C4) qui ne peuvent être mises en relation, contrairement à l'aqueduc $\mathrm{C} 6$, avec l'alimentation en eau des parties résidentielle et agricole de la villa situées à une centaine de mètres plus à l'ouest et au nordouest (cotes : $318,80 \mathrm{~m}$ à $317,16 \mathrm{~m} \mathrm{NGF}$ ).

\section{DONNÉES STRATIGRAPHIQUES ET GÉOARCHÉOLOGIQUES (VERSANT ORIENTAL, ZONE III)}

Les données stratigraphiques ont permis d'appréhender l'ensemble du site de Richeaume I sur la longue durée avec notamment la mise en place d'une topographie en terrasses de la villa (fig. 137). L'étagement des premières structures a été réalisé selon la pente naturelle du terrain avec, dès la fin du $\mathrm{I}^{\mathrm{er}}$ s. apr. J.-C., des nivellements successifs sur l'ensemble de l'éminence et sur les versants méridionaux et orientaux (pendages nord-sud et ouest-est du terrain). La réoccupation médiévale du site a également provoqué un remaniement des

110. Nous tenons à remercier tout particulièrement A. Badic, J.-L. Ballais et J.-M. Gassend (Institut de recherche de l'architecture antique, (NRS) qui effectuent depuis 1997, avec la collaboration de V. Dumas, les relevés topographiques de l'ensemble de ces structures et du site.

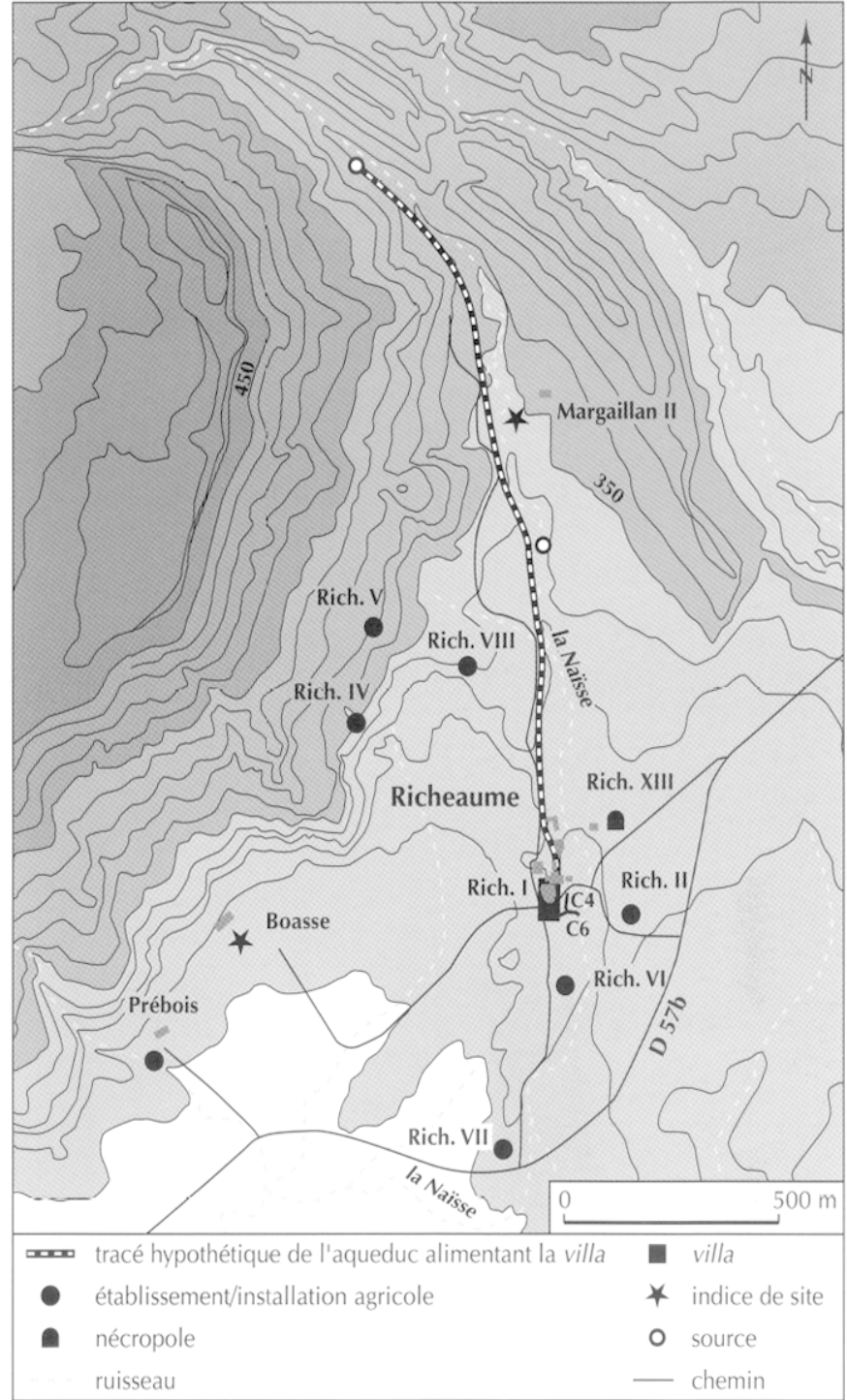

Fig. 135 - Carte archéologique des sites du Haut-Empire et de l'Antiquité tardive autour du site de Richeaume I el du tracé hypothétique de l'aqueduc alimentant la villa (DAO V. Dumas et F. Mocci, CNRS).

couches archéologiques les plus profondes notamment sur le versant oriental, dans la zone III (fossés, fosses, chemin).

Sur cette zone, au-delà des données purement archéologiques afférentes à la chronologie et à l'organisation du site, les sondages géoarchéologiques menés depuis 1999 ont permis de restituer l'évolution topographique du secteur (fig. $138 \mathrm{et}$ 139). Ils ont permis de repérer un cours d'eau antique (le paléochenal 13010) et de localiser un tronçon de l'aqueduc C4 sur ses deux rives (voir infra, p. 153-158). Ce paléochenal, qui suit bien un cours parallèle au ruisseau contemporain de la Naïsse, est composé de deux chenaux (fig. 139) : le plus ancien (LS 22006), dont seul le sommet du remplissage a été atteint dans le sondage (US 22007), est sans doute antérieur à l'occupation gallo-romaine. Le second (US 13010) s'est creusé dans les alluvions fines des crues du premier paléochenal (LS 22007). 


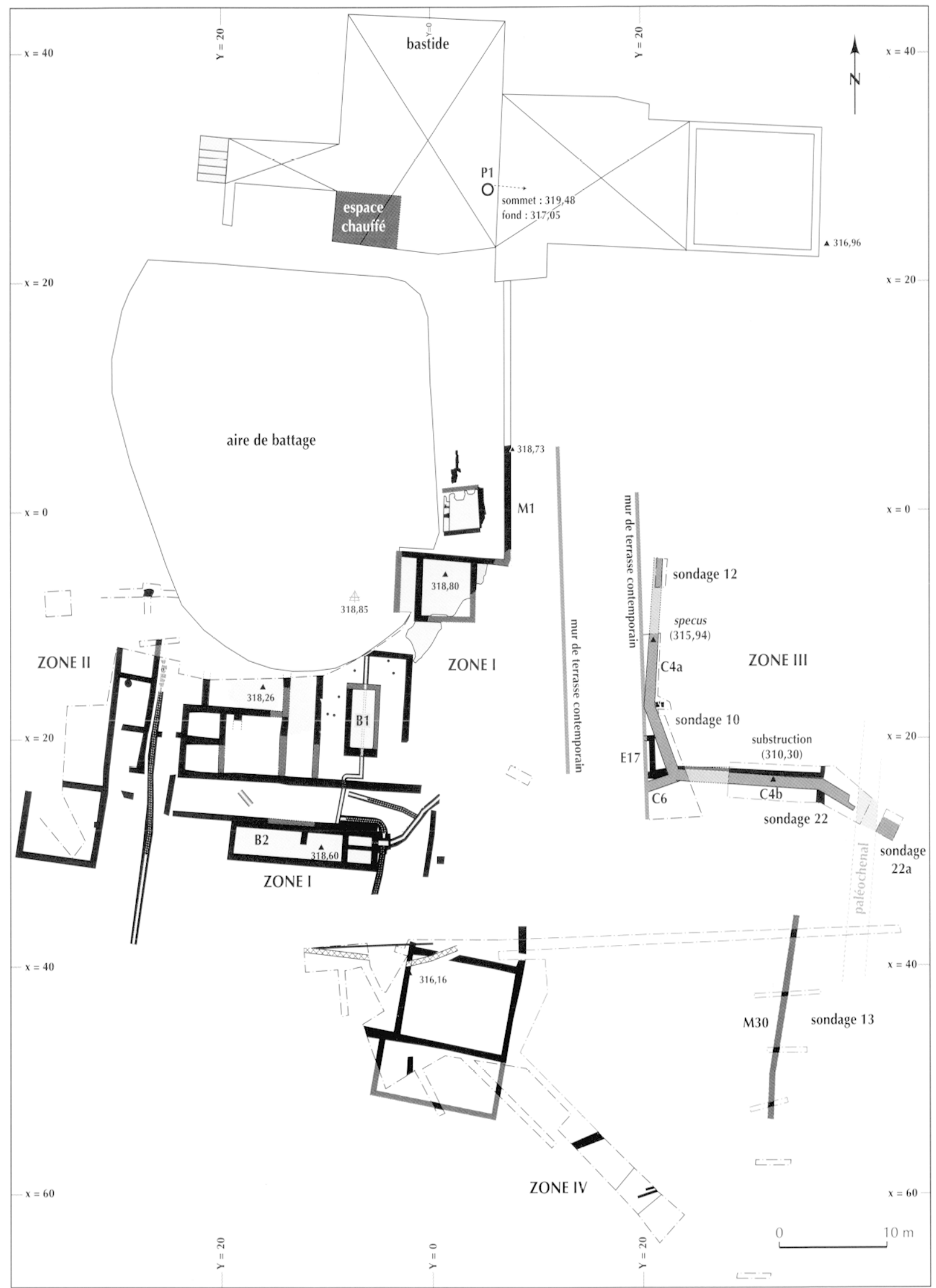

mur conservé en fondation ou en élevation $\square$ mur restitué $\square$ canalisations et bassins conservés $\square$ canalisations et bassins restitués

Fig. 136 - Plan général des vestiges archéologiques mis au jour sur le site de Richeaume I (relevés J.-M. Gassend, J.-L. Paillet, A. Badie et M.-L. Laharie, I)AO V. Dumas, (NRSS). 

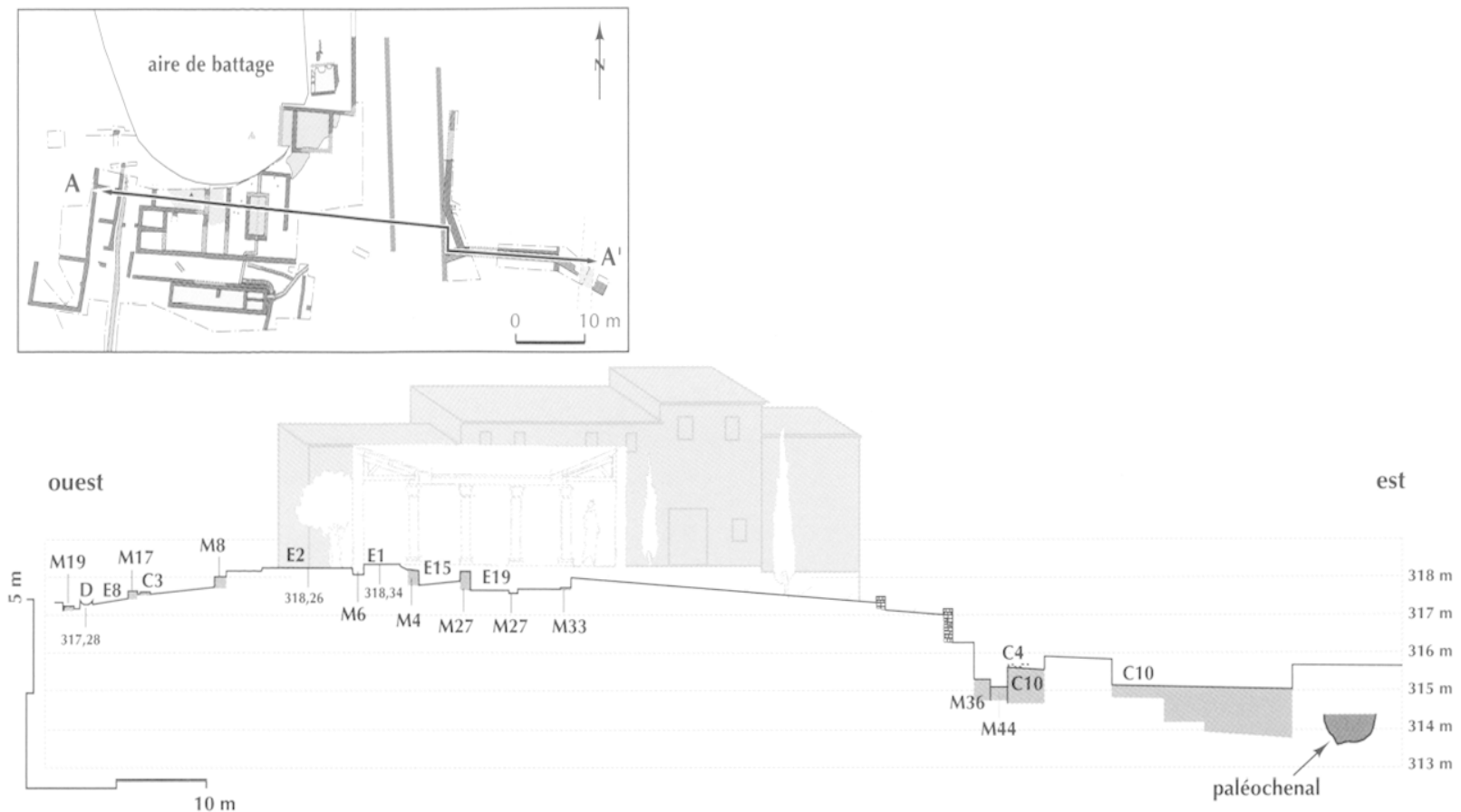

Fig. 137 - Coupe transversale des vestiges archéologiques du site de Richeaume I (DAO V. Dumas et J.M. Gassend, CNRS).

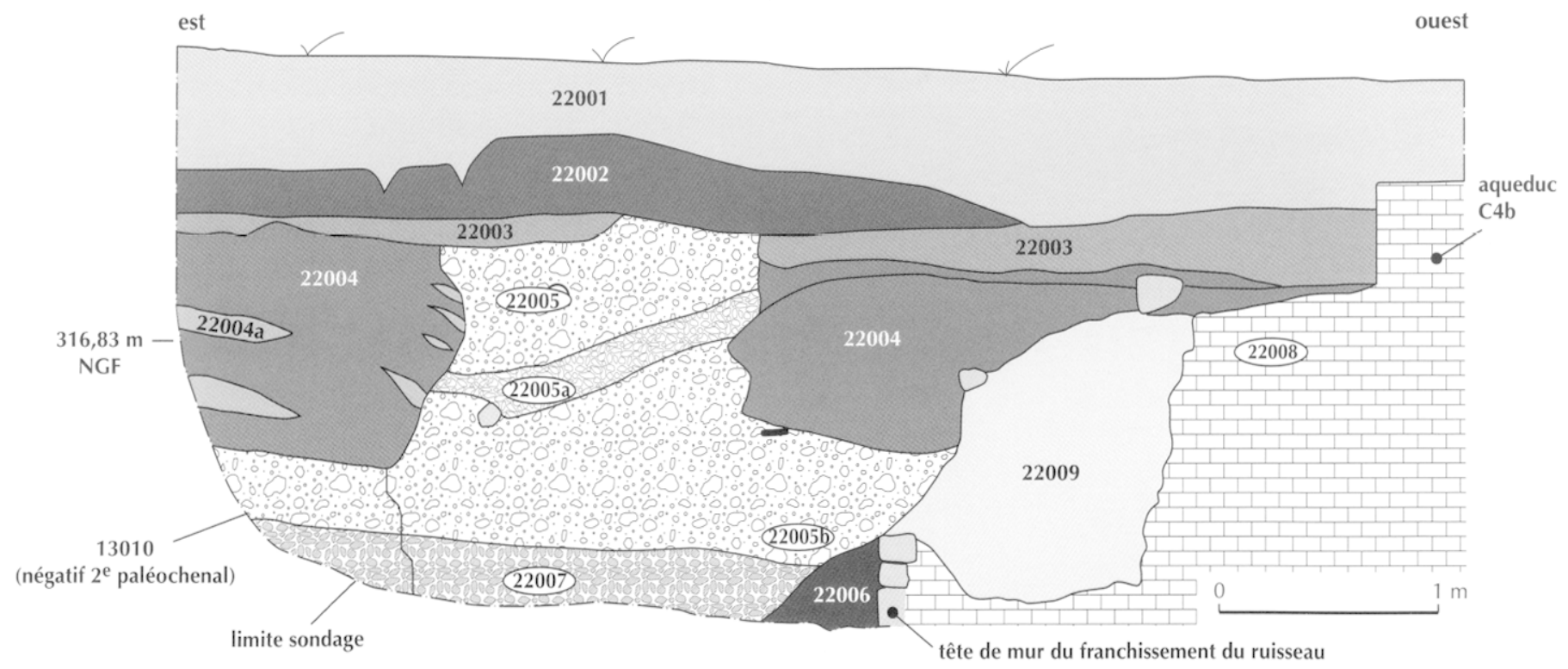

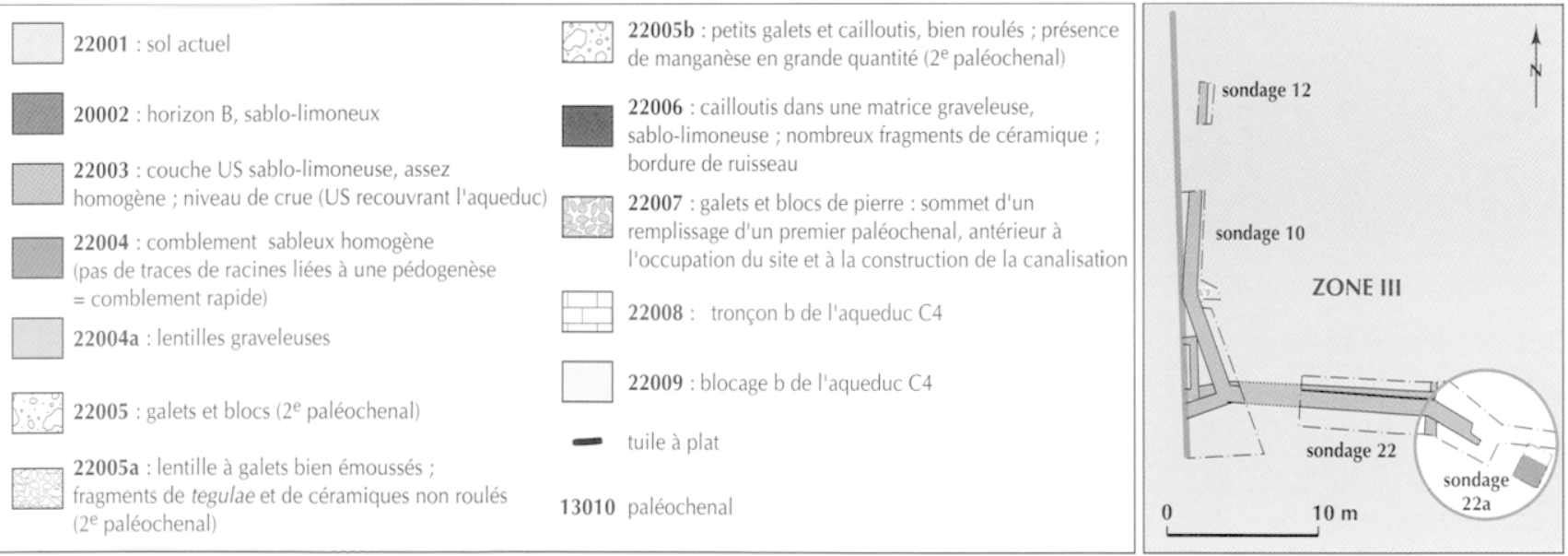

Fig. 138 - Coupe géoarchéologique du paléochenal 13010 et du tronson b de l'aqueduc C4 dans le sondage 22 (DAO V. Dumas, C. Miramont, CNRS et K. Walsh, université de York). 

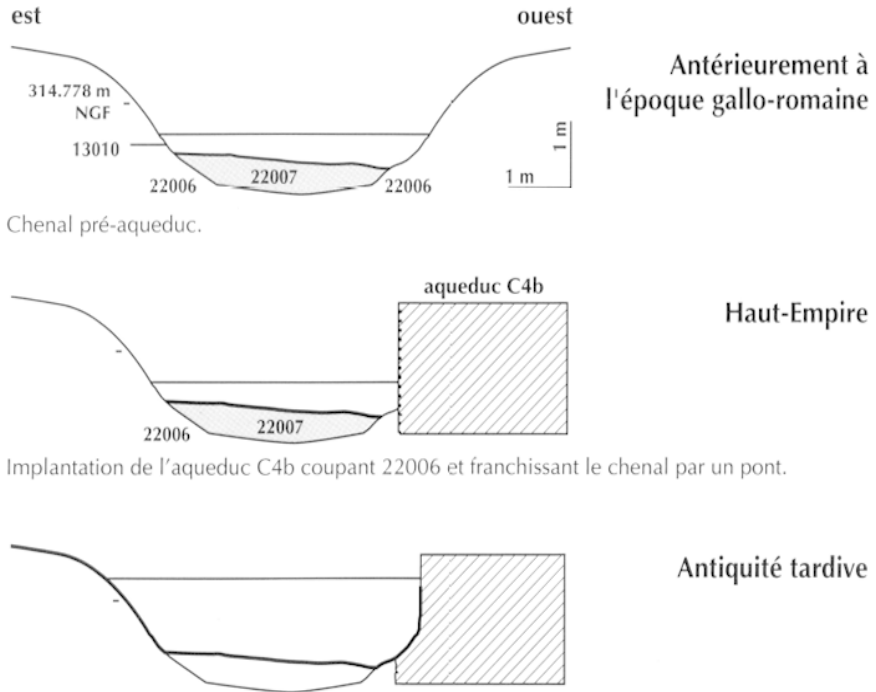

Antiquité tardive

L'extrémité est de l'aqueduc est recoupée par un nouveau chenal qui le détruit particllcment.

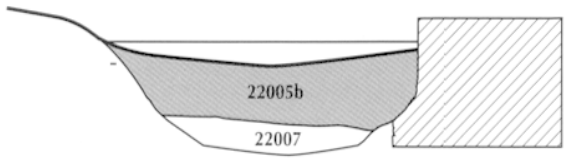

Antiquité tardive/ Haut Moyen Âge

Comblement du chenal par 22005b.

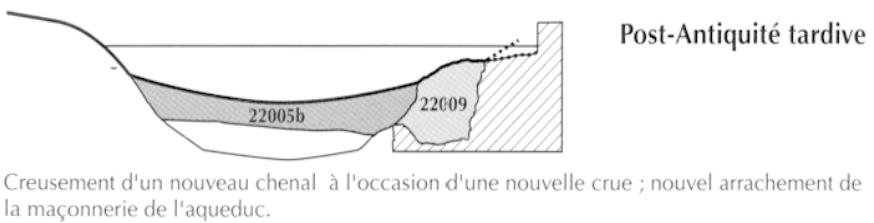

la maçonnerie de l'aqueduc.

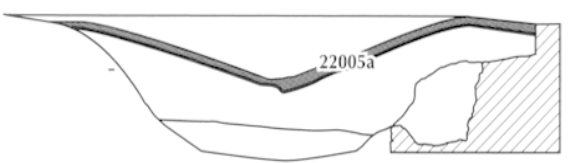

Période médiévale?

Nouvelle crue, creusement du chenal et comblement par 22005a.

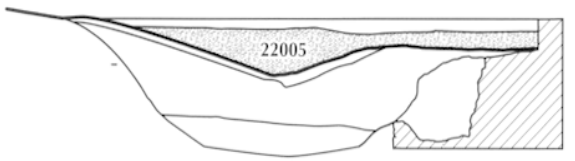

Période médiévale?

Crue importante entraînant une restructuration complète du chenal qui s'élargit en détruicant davantage la maçonnerie de l'aqueduc ; l'eau occupe toute la largeur du chenal et dépose des blocs et des cailloux (22005).

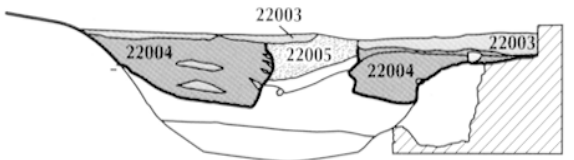

Période moderne?

Le débit ralentit, l'eau circule dans des chenaux restreints comblés par les sables (22004) puis par des limons (22003) au fur et à mesure que la force du courant diminue.

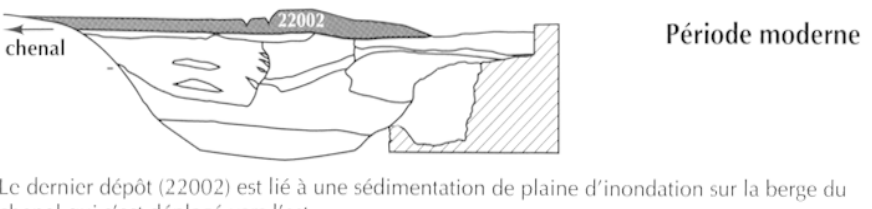

Lc dernier dépôt (22002) est lié à une sédimentation de plaine d'inondation sur la berge du chenal qui s'est déplacé vers l'est.
Fig. 139 - Essai de restitution des phases d'évolution du paléochenal 13010 et de l'extrémité est du troņ̧on b de l'aqueduc C4 (DAO C. Allinne, V. Dumas, CNRS et K. Walsh, université de York).

Cette première phase d'incision du chenal 13010 est sans doute contemporaine de l'occupation de la villa. En effet, ce bras de ruisseau devait être actif lors de la construction de l'aqueduc, comme en témoignent les vestiges de la tête de mur du franchissement de ce cours d'eau identifiée également sur la rive gauche du paléochenal (sondage 22a). Plusieurs séquences de creusement et de dépôt affectent ce paléochenal (US 22005b22005a-22005) et détruisent l'extrémité est de l'aqueduc, révélant ainsi les effets d'une phase d'érosion succédant à l'abandon du site antique que nous situons à l'Antiquité tardive ou au haut Moyen Âge ${ }^{111}$. Cette période d'érosion observée sur le site de Richeaume peut être rattachée à des phénomènes mis en évidence dans la région à la fin de l'Antiquité. En effet, elle pourrait correspondre à l'abandon de la gestion des terroirs et des versants plutôt qu'à une crise climatique; hypothèse qui était suggérée il y a quelques années. En analysant le bassin versant de la Naïsse, nous constatons la présence de versants décapés sur lesquels apparaît une forte pédogenèse antérieure et postérieure à l'Antiquité. Les couches sédimentaires US 22004, 22003 et 22002 représentent les crues les plus récentes de la Naïsse (périodes moderne puis contemporaine).

Plus généralement, les données géoarchéologiques confirment une rupture de pente très prononcée, identifiée au sud et à l'est de la zone d'habitat de la villa et soulignée par le mur de clôture nord-sud M1 (fig. 136). Elles révèlent également un colmatage sédimentaire de ce secteur dès le début de l'Antiquité, mais surtout à l'époque moderne, par des phases successives de débordement et de crues liées à la dynamique fluviale ainsi que des périodes d'érosion: sédimentation de pente, colluvionnement. L'aménagement des versants méridionaux et orientaux par des murs de terrasses et de digues (voir infra M53-M30, p. 156-157) témoigne de ces phénomènes et conjugue un certain nombre d'avantages, notamment face aux crues du ruisseau, comme l'atteste la présence de drains antiques et médiévaux.

Dans l'état actuel des recherches, alors que le Haut-Empire serait caractérisé par une tendance à l'incision lente des talwegs (Ballais, Crambes, 1992), l'Antiquité tardive correspondrait à quelques remblaiements localisés, peu épais et discontinus, mais qui reflètent des épisodes d'écoulements à caractère torrentiel ${ }^{112}$. La période moderne, plutôt caractérisée par l'absence ou la très faible épaisseur des dépôts, correspond à un aménagement intensif de terrasses de cultures qui piègent la charge solide potentielle sur les versants. Il parait donc difficile d'évoquer une « crise environnementale " sur ce secteur pour

111. Il est antérieur à une nappe colluviale dont la base est datéc par le ${ }^{1.4}$ : entre 1290 et 1571 apr. J.-C. sur le secteur de Margaillan, situé à $1 \mathrm{~km}$ en amont de Richeaume I (Jorda, Provansal, 1992).

112. Au pied de l'oppidum de Roque-Vaoutade sur le versant méridional de la montagne de la Sainte-Victoire, l'un d'eux est daté par le ${ }^{1.1}$ C: entre 4.38 et 660 apr. J-C. 
la période considérée, malgré des facteurs plutôt favorables à l'érosion (pentes, lithologie). Les indices d'épisodes érosifs, très localisés, reflètent en partie l'état des recherches encore incomplet ; néanmoins nous pouvons postuler que ces épisodes ne sont pas liés à une surexploitation des terroirs. Les phases d'érosion antérieures (Pléistocène et Holocène) ont fait disparaître l'essentiel des couvertures mobilisables, expliquant la minceur des dépôts historiques (Leveau et al., 1992 ; Walsh, Mocci, 2003).

\section{LES AMÉNAGEMENTS HYDRAULIQUES DU VERSANT ORIENTAL DE LA VILLA}

Les aménagements hydrauliques reconnus dans la zone III s'inscrivent au sein d'une vaste villa, en pleine expansion entre la première moitié du $\mathrm{II}^{e} \mathbf{s}$. (état IIIa) jusqu'à la fin du IV ${ }^{\mathrm{e}} \mathrm{s}$. apr. J.-C. (état $\mathrm{Vb})^{113}$. À la fin du $\mathrm{I}^{\mathrm{er}}$ s. de notre ère (état IIIa), alors que la partie résidentielle d'une première villa s'organise sur la zone centrale de l'éminence (zone I), autour d'une cour avec péristyle et bassin d'agrément (B1), le versant oriental du plateau est aménagé en terrasses à l'est du mur de clôture M1 (zone III) et un très long mur de digue nord-sud est élevé (M53-M30). Ln aqueduc (C4) est alors construit contre un bâtiment antérieur ${ }^{114}$ (E17) afin, sans doute, d'alimenter l'une des installations agricoles situées en aval de la villa ${ }^{115}$. Dans la seconde moitié du IV $\mathrm{s}$. (état $\mathrm{Vb}$ ), en relation avec des réaménagements plus importants réalisés sur l'ensemble du site ${ }^{116}$, une nouvelle branche d'aqueduc (C6) est construite à partir de l'aqueduc C4 (fig. 140 et 141). Dans le premier quart du ve s. (état VI), les aqueducs C4, C6 et le mur de digue M53 ne sont plus en fonctionnement (Mocci, 2000 et 2001).

\section{LES VESTIGES DE L'AQUEDUC C4}

Mis au jour sur une longueur de $26,68 \mathrm{~m}$ et $28,33 \mathrm{~m}$ et sur une hauteur comprise entre $0,50 \mathrm{~m}$ et $1,95 \mathrm{~m}$, les vestiges de l'aqueduc C4 correspondent à un imposant mur porteur de

113. Les phases majeures d'occupation et de réaménagement de la villa correspondent, à ce jour, à l'état II ( $\mathrm{I}^{\mathrm{er}}$ s. av. J.-C.-fin du $\mathrm{I}^{\mathrm{er}}$ s. apr. J.-C.), à l'état III (IIIa: fin du I ${ }^{\text {er }}$ s.- $1^{\text {re }}$ moitié du II $^{\mathrm{e}}$ s. et IIIb : $1^{\text {re }}$ moitié du $\mathrm{II}^{\mathrm{e}} \mathrm{s}$-début du $\mathrm{III}^{\mathrm{e}} \mathrm{s}$.), à l'état $\mathrm{VV}$ ( $\mathrm{I}^{\mathrm{re}}$ moitié du $\mathrm{III}^{\mathrm{e}} \mathrm{s}$-début du $\mathrm{I}^{\mathrm{e}} \mathrm{s}$.) et à l'état $\mathrm{Vb}\left(1^{\text {re }}\right.$ moitié du $\mathrm{IV}^{\mathrm{e}} \mathrm{s}$.-1 ${ }^{\text {cr }}$ quart du $\mathrm{v}^{\mathrm{re}} \mathrm{s}$.).

114. Ce bâtiment, construit au cours du I ${ }^{\text {er }}$ s. apr. J.-C. (état II), n'a pu être que partiellement mis au jour. Il a été construit en partie sur les extrémités orientale et méridionale de la margelle d'un puits du $\mathrm{I}^{\mathrm{er}} \mathrm{s}$. av. J.C. (US $10049: 1,32 \mathrm{~m}$ de diamètre). Seules cinq assises, soit $0,74 \mathrm{~m}$ de hauteur, ont pu être dégagées (profondeur atteinte: cote $314,48 \mathrm{~m}$. NGF).

115. Richeaume II, localisé en rive gauche du cours actuel de la Naïsse ou Richeaume VI, situé plus au sud sur la rive droite de la Naisse (voir infra et fig. 142).

116. Dans la partie résidentielle: extension de l'habitat vers le sud (zones II et IV), construction de canalisations et transformation du long bassin B2 en cure agricole (zone I); dans la partie agricole: construction de nourelles pièces et d'une longue canalisation d'éracuation des eaux (C3).
1,10 m de large en petit appareil très soigné (opus vittatum), lié au mortier et aux joints soulignés d'un trait tiré au fer (fig. 142). Au sommet de cette substruction, un canal de circulation des eaux (specus) a été partiellement conservé sur le tronçon C4a seulement (fig. 141 et 142). L'une des caractéristiques de cet ouvrage provient de son tracé non rectiligne que nous avons distingué en deux tronçons ( $\mathrm{C} 4 \mathrm{a}$ et $\mathrm{C} 4 \mathrm{~b}$ ), identifiés dans les sondages 10,12 et $22^{117}$ : le tronçon C4a est orienté nord-sud sur $8,75 \mathrm{~m}$ de long, puis diverge vers le sud-est afin de contourner le bâtiment E17 sur $5,35 \mathrm{~m}$ de long. De là, le tronçon $\mathrm{C} 4 \mathrm{~b}$ s'oriente $\mathrm{N} 70^{\circ}$ vers l'est sur une dizaine de mètres, puis vers le sud-est au-delà du cours d'eau 13010 .

\section{LE TRONÇON A DE L'AQUEDUC C4}

Ce tronçon a été reconnu sur 14,10 m de long, selon un axe nord-sud (sondages 10 et 12). Les fondations de cet ouvrage, constituées d'une ou deux assises de gros blocs partiellement équarris et liés au mortier (de 13 à $23 \mathrm{~cm}$ de haut), reposent sur le substrat calcaire (argilites, US 10054, cotes NGF : 314,88 m à l'est et $315,15 \mathrm{~m}$ à l'ouest). Le parement oriental du mur porteur, conservé sur une hauteur de $38 \mathrm{~cm}$ à $43,50 \mathrm{~cm}$, est composé de cinq assises en opus vittatum. Le specus est formé d'un conduit de maçonnerie en béton de tuileau (larg. : $21 \mathrm{~cm}$; ép. : 13,5 cm) avec bourrelets d'étanchéité (ép. : $8 \mathrm{~cm}$; haut. : $7 \mathrm{~cm}$ ), bordés à l'ouest et à l'est, par un piédroit en petit appareil (fig. 142) : le piédroit ouest mesure $35 \mathrm{~cm}$ de large et est conservé sur deux assises, soit $23 \mathrm{~cm}$ de haut ; celui de l'est, d'une largeur de $30 \mathrm{~cm}$, possède une à quatre assises à son extrémité sud (haut. 10 à $41 \mathrm{~cm}$ ). Ces murs bajoyers sont constitués de petits moellons équarris et de tegulae enduites de mortier de tuileau. Le fond du specus comportant de rares traces de dépôts carbonatés (cote moyenne : 315,94 m NGF) n'est relativement bien conservé que sur $8 \mathrm{~m}$ de long (fig. 141, $\mathrm{n}^{\circ} 1$ ) : au-delà, il a été détruit par deux fossés médiévaux parallèles de direction nord-est - sud-ouest.

La couverture de ce canal n'a pas été conservée, mais elle était probablement constituée de grandes dalles de grès plates et de tegulae, telles celles découvertes dans des couches de destruction : US 10015 et 10020 (fig. 140).

\section{LE TRONÇON B DE L'AQUEDUC C4}

Mis au jour dans les sondages 10 et 22, le tronçon $\mathrm{C} 4 \mathrm{~b}$ a été reconnu sur une longueur totale de $12,58 \mathrm{~m}$ à 14,23 m. Dans le sondage $10^{118}$, le parement nord possède deux assises de fondation sur $13 \mathrm{~cm}$ de hauteur, élevées sur le substrat calcaire ; le parement sud est constitué de cinq à sept assises (de 42 à $70 \mathrm{~cm}$ de haut) reposant directement sur une couche de remblai (US 10058) et à son extrémité ouest, sur une construction antérieure (US 10065). Le specus de ce tronçon a été détruit par le fossé médiéval C8.

117. La jonction entre ces sondages n'a pu être réalisée en raison de la présence d'arbres (ces vestiges sont situés en plein cœur du jardin de la propriété actuelle).

118. Dans ce sondage, il a été mis au jour sur unc longucur de $0,68 \mathrm{~m}$ (parement nord) à $1,13 \mathrm{~m}$ (parement sud). 


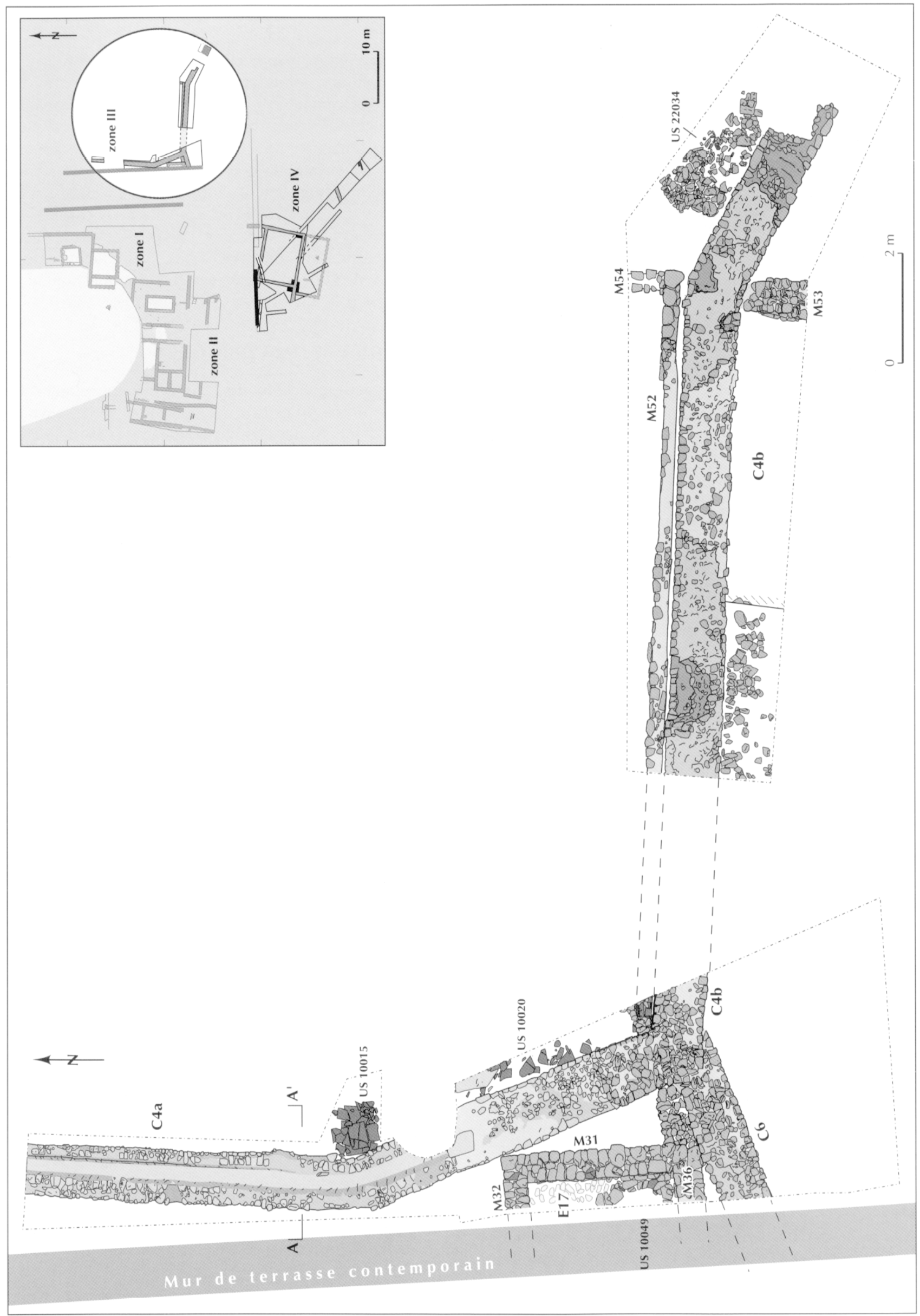

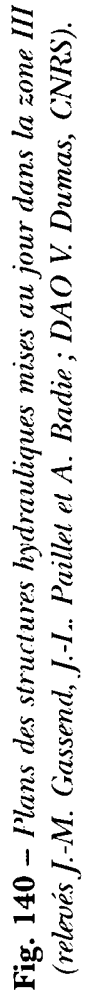




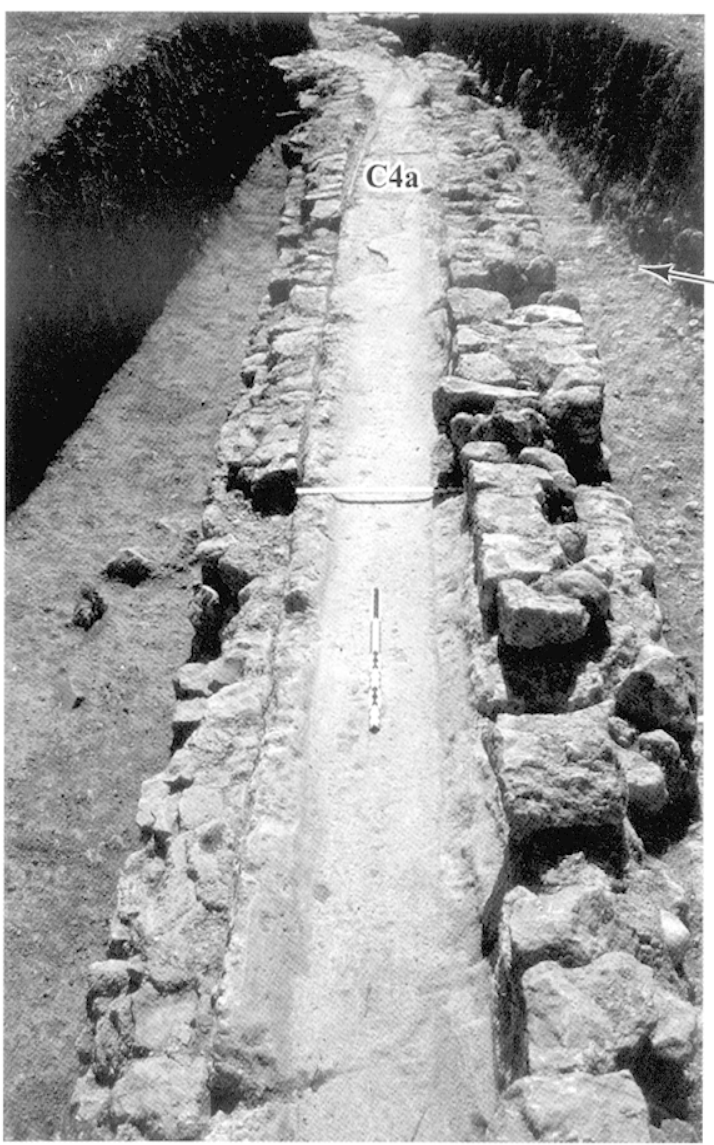

1

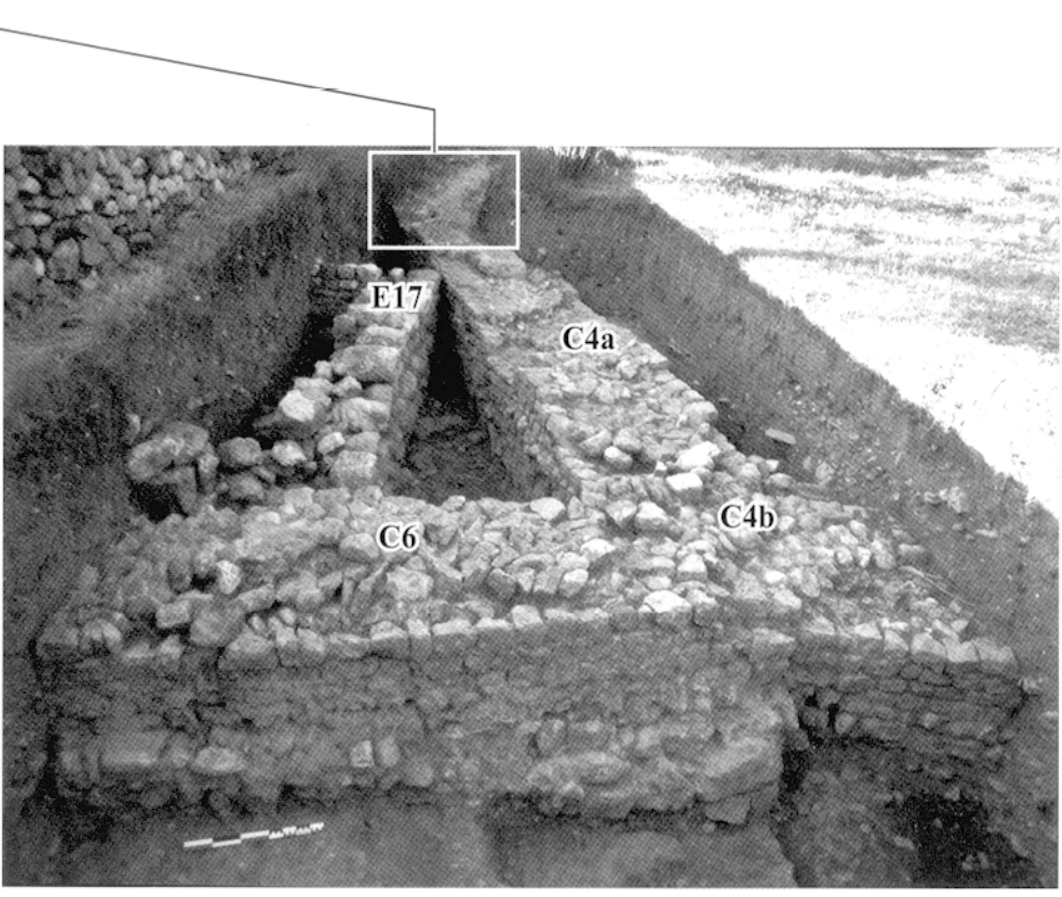

2

Fig. 141 - Sondage 10: 1, vue depuis le nord du specus du tronçon b de l'aqueduc C4 ; 2, vue depuis le sud des aqueducs C4 et C6, et du bâtiment E17 (cliché F. Mocci, CNRS).
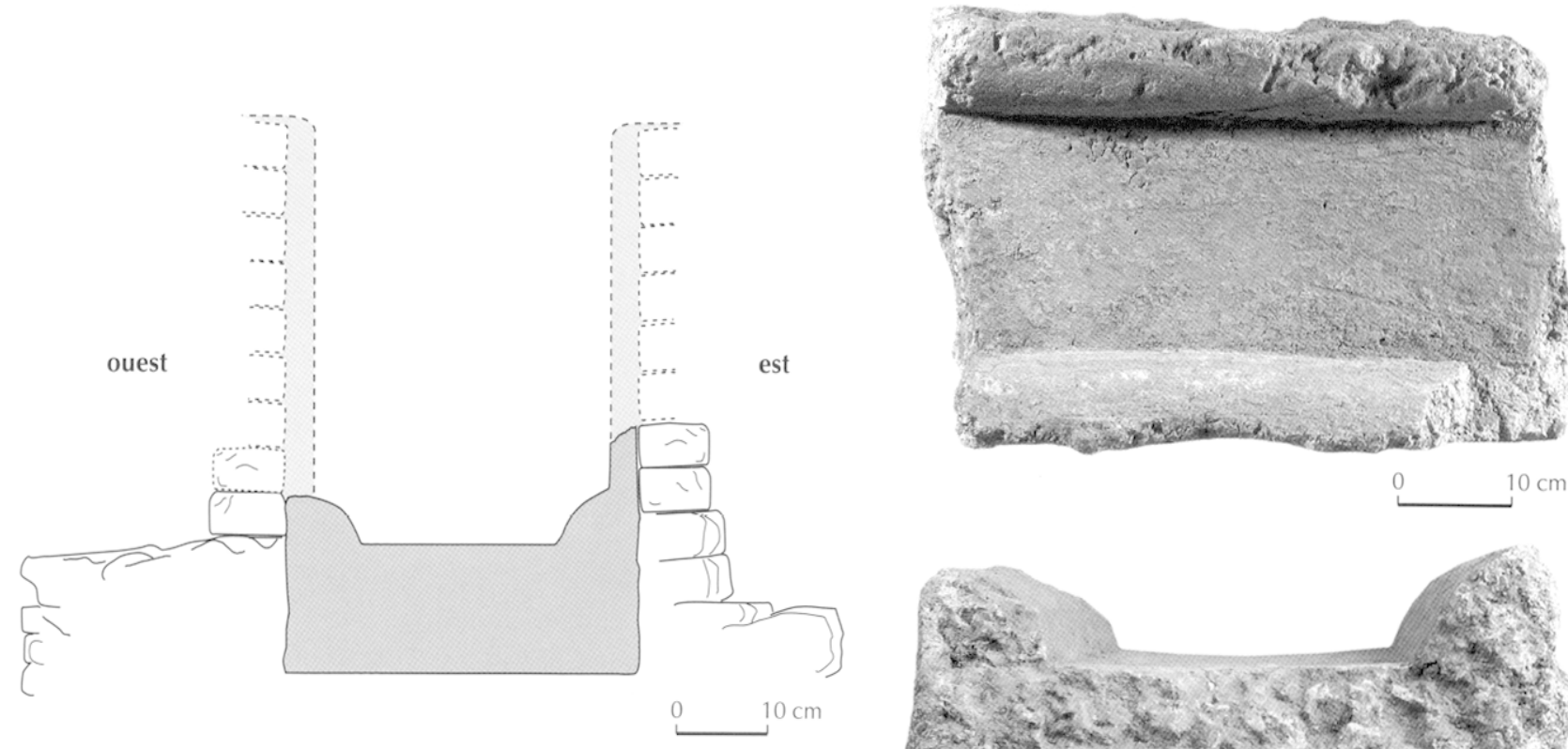

Coupe $\mathrm{A}-\mathrm{A}^{\prime}$

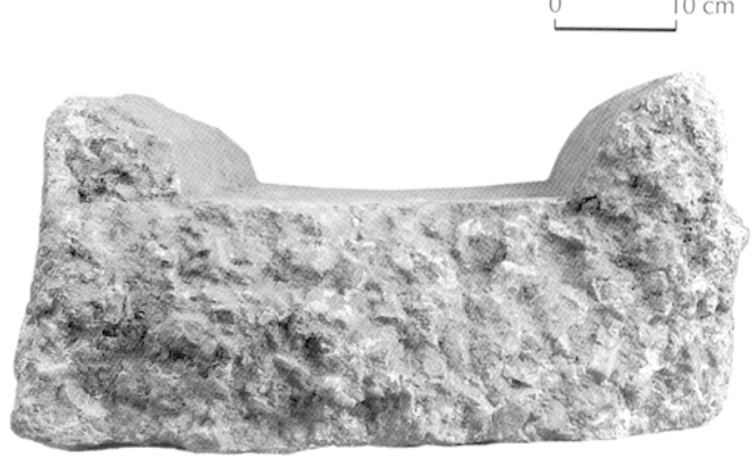

0

$10 \mathrm{~cm}$

Fig. 142 - Section du specus du tronçon a de l'aqueduc C4 et photographies d'une portion du fond du specus réutilisée dans un mur de terrasse médiévale (clichés C. Durand, CNRS). 


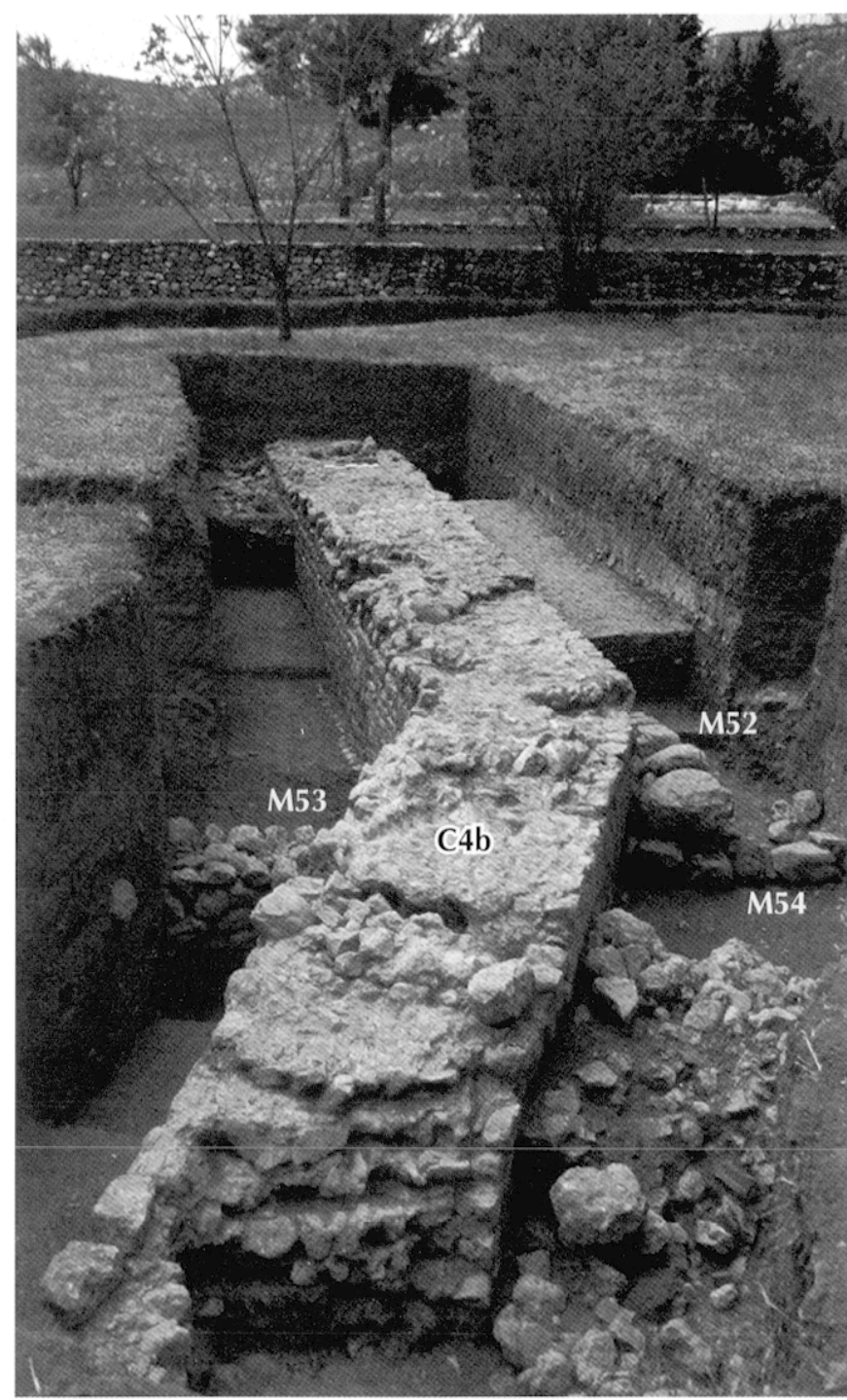

Fig. 143 - Vue depuis le sud-est du tronçon b de l'aqueduc C4 et des murs M52, M53 et M54 dans le sondage $22 ; D 4$, à l'arrière-plan, bâtiments de la villa sur les terrasses supérieures (cliché F. Mocci, CNRS).

Dans le sondage 22 (fig. 141, $\mathrm{n}^{\circ} 2$, et fig. 142 et 143), seul subsiste le mur de soutènement reconnu sur $11,90 \mathrm{~m}$ et $13,10 \mathrm{~m}$ de long (cotes sommet : entre $315,04 \mathrm{~m}$ et $314,07 \mathrm{~m} \mathrm{NGF).} \mathrm{De}$ direction ouest-est sur $9 \mathrm{~m}$ de long, cette substruction bifurque brusquement vers le sud-est (fig. 143). Depuis ce changement d'orientation ${ }^{119}$, seuls $2,90 \mathrm{~m}$ le long du parement nord et 4,10 m le long du parement sud de cet ouvrage sont conservés. Au-delà, après le franchissement du cours d'eau 13010, il a été reconnu sur $1 \mathrm{~m}$ de long seulement (sondage $22 \mathrm{a}$ ) ${ }^{120}$. Les

119. Ce changement d'orientation pourrait être lié soit à une rupture de pente, soit à la topographie du secteur. La perspective de franchissement du cours d'eau de manière à atteindre la berge opposée pourrait être aussi envisagée.

120. Les sondages réalisés au cours du mois de septembre 2003 en rive gauche de ce cours d'eau, en direction du sud et de l'est, n'ont pas permis de localiser le tracé en aval de cet aqueduc. parements, particulièrement bien conservés, ont été dégagés entre $0,32 \mathrm{~m}$ et $1,59 \mathrm{~m}$ de profondeur (fig. 144). Ils soulignent la forte déclivité et la profondeur du substrat sur lequel ils reposent. Une à neuf assises ont été mises au jour le long du parement nord (soit entre $0,12 \mathrm{~cm}$ et $0,89 \mathrm{~cm}$ de haut), la fondation n'ayant pas été atteinte. Deux à treize assises ont été dégagées le long du parement sud (soit entre 0,32 $\mathrm{m}$ et $1,05 \mathrm{~m}$ ) ainsi qu'une à trois assises de fondation (petits blocs non équarris) soit $0,10 \mathrm{~m}$ et $0,30 \mathrm{~m}$ de haut.

L'extrémité orientale de la canalisation, dégagée sur huit assises (90 cm de haut), a été détruite en grande partie par les débordements du cours d'eau 13010, sans doute au cours du $\mathrm{V}^{\mathrm{e}}$ s. apr. J.-C. (fig. 138 et 139). En raison d'une profondeur trop importante et des recouvrements sédimentaires, la base des assises de fondation n'a été que partiellement identifiée mais elle témoigne de l'existence d'un pont pour le passage du cours d'eau. Un changement de facture dans la construction de la canalisation, visible en amont d'une rupture partielle de ce tronçon à 2,50 m environ de l'extrémité est, correspond sans doute à une phase de réfection de la canalisation; cette nouvelle construction, trop fragile, n'a pas résisté aux crues ou à une élévation du niveau du cours d'eau. En aval de cette rupture, le parement sud, constitué de gros blocs de pierre au lieu de moellons équarris et réguliers, est recouvert par d'importants dépôts carbonatés sur 2,40 $\mathrm{m}$ de long (fuites de l'ouvrage ?). Le parement nord, de même facture sur 1,84 $\mathrm{m}$ de long, ne possède pas de concrétions.

De part et d'autre du mur de soutènement de l'aqueduc, trois structures ont été partiellement dégagées (fig. 140, 141, $\mathrm{n}^{\circ} 2$ et fig. 143). Elles correspondent à un muret bordant le parement nord du tronçon C4b (M52 et M54) et à un mur perpendiculaire au parement sud (M53).

\section{Les murets M52 et M54}

Le muret M52 (cote : $315,12 \mathrm{~m} \mathrm{NGF)} \mathrm{a} \mathrm{sans} \mathrm{doute} \mathrm{été}$ construit après le tronçon $\mathrm{C} 4 \mathrm{~b}$ dont il borde le parement nord à une distance de 6 à $14 \mathrm{~cm}$ (sondages 10 et 22) (fig. 140 à 145). Ce muret, dont la fonction n'a pu être définie, comprend probablement deux phases de construction : la première, mise au jour à l'extrémité est sur 1,78 m de long, correspond à un muret de $35 \mathrm{~cm}$ de large constitué de trois assises de gros blocs de pierre non taillés, entre lesquels s'intercalent des tegulae posées à plat et du mortier (cote sommet : $314,71 \mathrm{~m} \mathrm{NGF).} \mathrm{La}$ seconde phase correspond à l'élévation sur le sommet de la troisième assise d'un muret de $30 \mathrm{~cm}$ de large construit avec des moellons non équarris liés au mortier (sommet du M52). Le premier état de construction de ce mur (état II ?) pourrait être associé au mur M54 situé à l'extrémité est du mur M52, perpendiculairement à l'aqueduc C4. Reconnu sur une longueur de 1,20 m et une largeur de $60 \mathrm{~cm}$, ce mur M54 conserve deux assises (parement est) de même facture que le premier état du mur M52 (cote sommet : 314,52 m NGF). Il repose sur une couche de remblai dont la datation n'a pas pu être établie.

\section{Le mur M53}

Le mur M53 s'inscrit dans le prolongement septentrional du mur M30 mis au jour sur une vingtaine de mètres dans la 


\section{PAREMENT NORD}

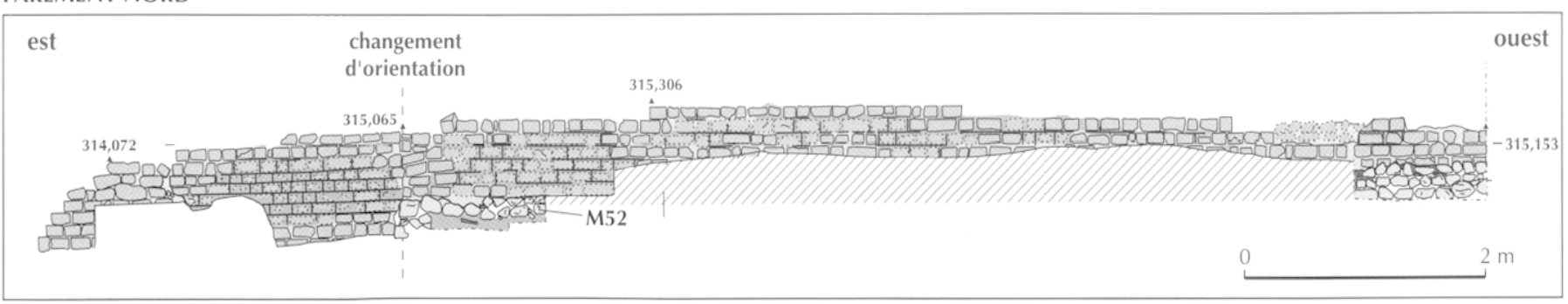

a

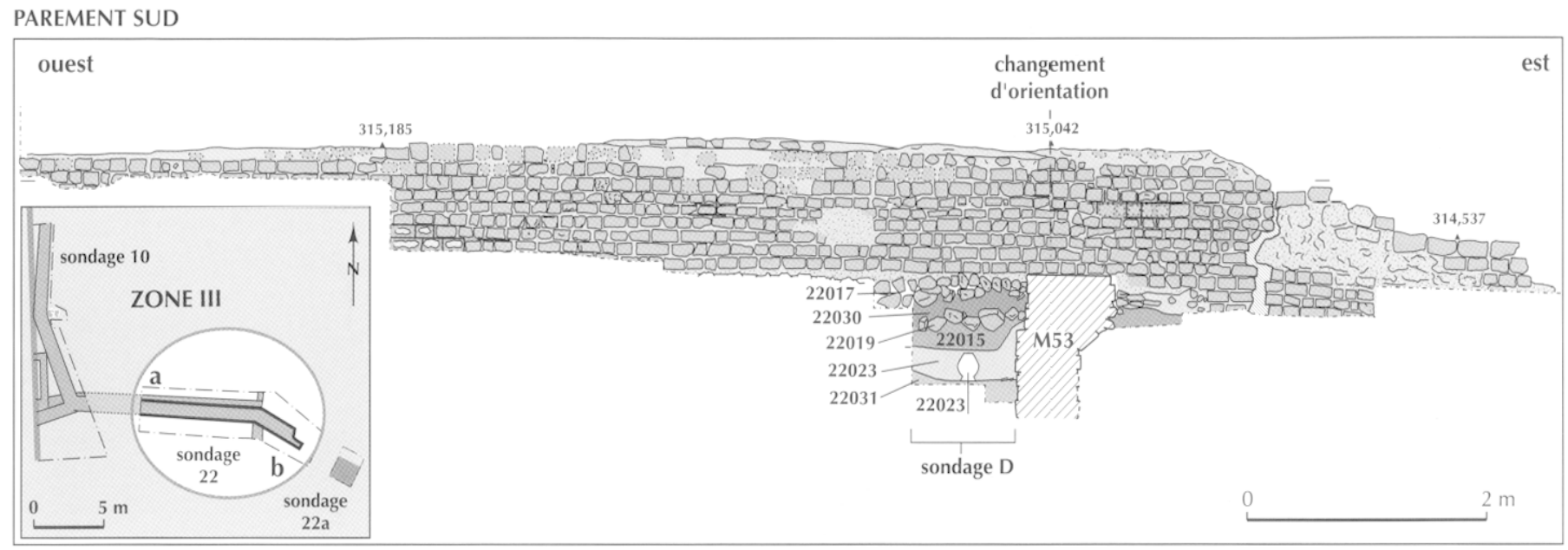

b

Fig. 144 - Coupes des parements nord (a) et sud (b) du tronson b de l'aqueduc C4 dans le sondage 22 (DAO V. Dumas, CNRS).

zone IV (fig. 140, 143 et 144b). Le parement sud du tronçon C4b repose sur l'extrémité nord de ce mur. Large de $60 \mathrm{~cm}$, il est constitué de gros blocs de pierre non équarris sans liant et de fragments de tegulae posés à plat (cote sommet: $314,05 \mathrm{~m}$ NGF). Le parement ouest conserve dix assises sur une hauteur totale de $1,07 \mathrm{~m}$; de petits blocs constituent les trois premières assises qui reposent sur une couche limono-sableuse (US 22031). Le parement oriental, fortement déversé, est constitué de six assises (cote base : $313,47 \mathrm{~m} \mathrm{NGF}$ ). Cette structure M53 faisait sans doute office de digue, témoignant ainsi d'un aménagement de la plaine dès le début du HautEmpire (fin état II-début état IIIa), suivant la pente du sol naturel, d'ouest en est. Une phase de débordement contemporaine du fonctionnement de ce mur conserve des fragments de céramique sigillée sud-gauloise Drag. 27 et des fragments d'amphore de Bétique. Ce niveau a été décapé par une phase de crue ultérieure dont il est difficile de préciser la datation. (début du Vtes. apr. J.-C. ?).

\section{DONNÉES STRATIGRAPHIQUES ET ÉLÉMENTS DE DATATION}

Un sondage réalisé contre le parement sud du tronçon $C 4 \mathrm{~b}$ a permis d'obtenir une séquence stratigraphique complète de sa construction et de celle du mur M53 bien en dessous des premières assises de fondation de l'aqueduc (US 22017) (fig. 137 et 144b). Une seule assise de blocs non équarris sans liant (US 22019), accolée contre le parement est du mur M53, a été ainsi mise au jour sous le tronçon $C 4 b$ et le remblai de nivellement US 22030. Il est difficile, dans l'état actuel de nos connaissances, de déterminer si cette structure est directement liée à la construction de l'aqueduc C.4 ou si elle lui est antérieure.

Sous ce niveau de blocs de pierre et sous une couche de remblai (US 22015) fut découvert dans l'US 22023, un vase sans anse intact, fermé, en céramique communc à pâte clairc (US 22025). D'une hauteur de $21 \mathrm{~cm}$, il était posé à l'envers, à plat, à $18 \mathrm{~cm}$ de profondeur depuis la base de l'LS 22019 et à $42 \mathrm{~cm}$ de profondeur de la base de la première assise de fondation du tronçon $\mathrm{C} 4 \mathrm{~b}$ (cote: $313,43 \mathrm{~m}$ NGF). Son couvercle, muni d'un bouton de préhension, était scellé par une couche d'argile. Ce vase pourrait être assimilé à un rite votif en liaison avec les aménagements hydrauliques du secteur; la fouille de la cour de l'archevêché à Aix-en-Provence avait révélé la présence, sous le sol de l'insula I, de vases de même type placés dans une position identique. Le terminus ante quem proposé pour la période d'activité de l'officine de potier se situait vers les années 80-90 apr. J.-C. (Rivet, 1986, p. 205-212).

L'analyse stratigraphique a permis de déterminer plus précisément les phases de fonctionnement puis de destruction 


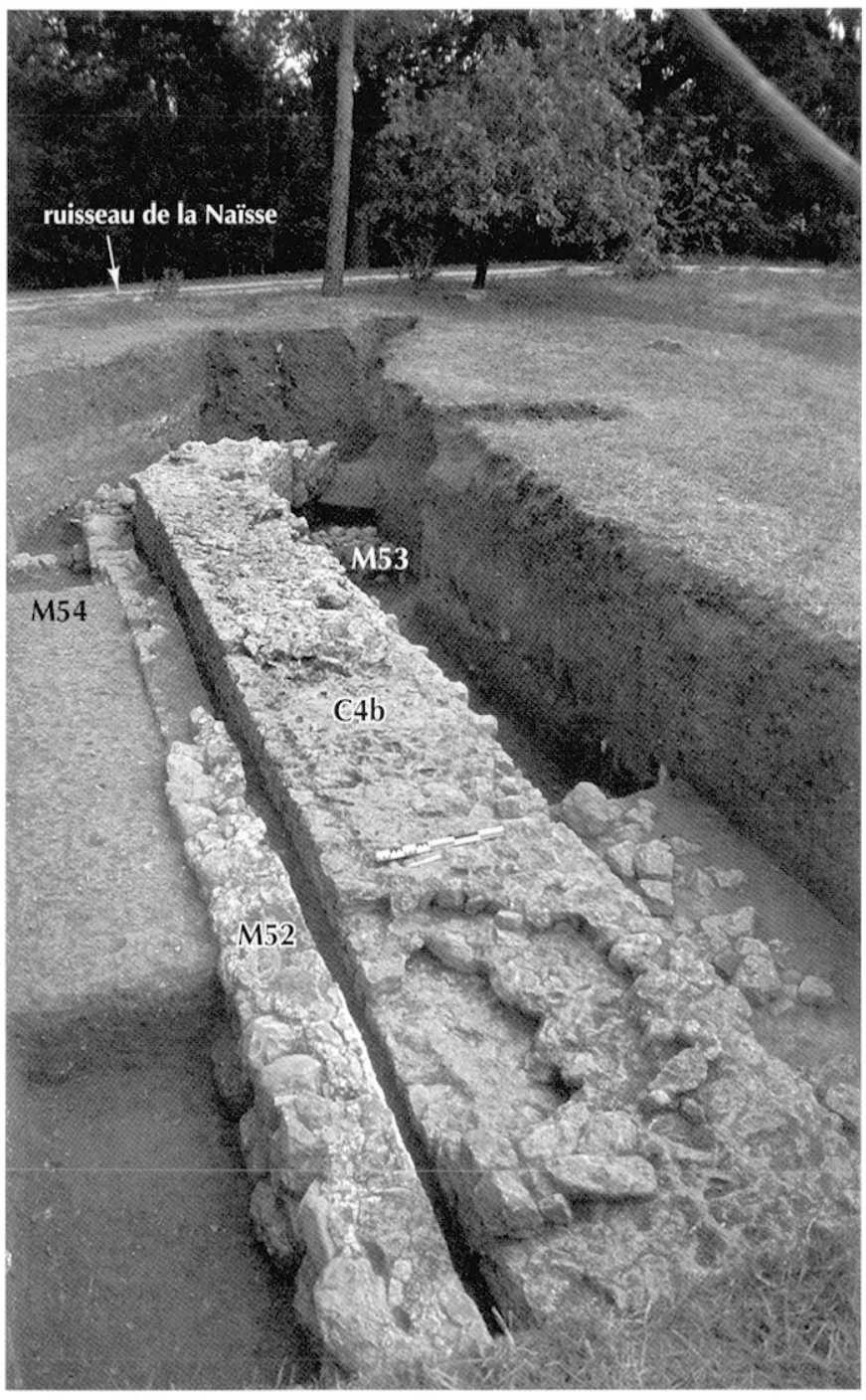

Fig. 145 - Vue depuis le nord-ouest du tronşon b de l'aqueduc C4 et des murs M52, M53 et M54 dans le sondage 22 (cliché F. Mocci, CNRS).

de l'aqueduc $\mathrm{C} 4$, ainsi que l'activité hydrologique liée à la présence du paléochenal 13010 et les phénomènes d'érosion du versant est. Les niveaux contemporains de l'utilisation de cet ouvrage C10 (état IIIa : $1^{\text {rc }}$ moitié du II ${ }^{\mathrm{e}} \mathrm{s}$. apr. J.-C..) correspondent à des remblais de fondation, à une phase de débordement du paléochenal 13010 et à un sol d'occupation dont le terminus ante quem se situe à la fin du $\mathrm{II}^{\mathrm{C}} \mathrm{s}$. apr. J.-C. Enfin, entre la fin de l'Antiquité et la période contemporaine, ont été identifiés des niveaux de destruction de cet ouvrage, tardifs ou modernes, une alternance de phases de débordement de la rivière et des périodes d'accalmie, avec remblais ou aménagements médiévaux et modernes recouverts par des niveaux de crues.

\section{LES VESTIGES DE L'AQUEDUC C6}

Construit contre les parements ouest et sud du tronçon b de l'aqueduc C4, sur une longueur de $43 \mathrm{~cm}$, les vestiges de l'aqueduc C6 correspondent à un tronçon du mur porteur en petit appareil lié au mortier d'une largeur de $0,90 \mathrm{~m}$ à $1 \mathrm{~m}$, de direction nord-est - sud-ouest (cotes NGF sommet est : $315,50 \mathrm{~m}$ et sommet ouest : $315,35 \mathrm{~m}$ ) (fig. 140 et $141, \mathrm{n}^{\circ} 2$ ). Le specus de cette canalisation a sans doute a été détruit par un fossé médiéval. Le parement nord de cette substruction a été mis au jour sur cinq à sept assises, soit une hauteur de $0,42 \mathrm{~m}$ à $0,70 \mathrm{~m}$; il repose sur le substrat calcaire mais aussi sur une des assises de l'angle interne du mur M36 de l'espace E17, alors épargné par l'aqueduc C4. Le parement sud est partiellement revêtu d'une couche de concrétions. Il a été mis au jour sur une hauteur de $0,97 \mathrm{~m}$ à $0,74 \mathrm{~m}$, sur neuf assises dont quatre assises de fondation en blocs non équarris (de $32 \mathrm{~cm}$ à $55 \mathrm{~cm}$ de haut). Il repose à son extrémité est sur la couche de remblai de fondation de l'aqueduc C4, mais aussi sur une construction antérieure contemporaine du bâtiment E17 (état II) ${ }^{121}$. La stratigraphie a permis de préciser la date de construction de la canalisation $\mathrm{C} 6$ au cours de la seconde moitié du $\mathrm{IV}^{\mathrm{e}} \mathrm{s}$. apr. J.-C. ${ }^{122}$.

Seul le départ de cet aqueduc a été mis au jour sur une longueur de 3,03 m (parement sud) et de 2,05 m (parement nord). Il démontre une dérivation (partielle ou totale ?) des eaux de l'aqueduc $\mathrm{C} 4$ vers la zone sud-ouest de la villa (installations agricoles en restructuration à cette même période).

Le terminus ante quem du fonctionnement des aqueducs $\mathrm{C} 4$ et C6 n'est pas clairement établi. En effet, les seuls éléments de datation ont été recueillis dans une couche de remblai des ouvrages de la fin du $\mathrm{IV}^{\mathrm{e}} \mathrm{s}$. apr. J.-C. (état $\mathrm{Vb}$ ). Plusieurs phases de destruction et d'aménagements (remblais, drains) visibles dans le sondage 10 interviennent au cours du ve s. et au début du VI ${ }^{\mathrm{e}}$ s. apr. J.-C. puis à partir du bas Moyen Âge.

$$
*
$$

En ce qui concerne les deux vestiges d'aqueducs présentés ici, le tronçon de l'aqueduc C6 est celui dont l'utilisation semble relativement claire, sa construction, depuis l'aqueduc C4, étant probablement liée à l'alimentation en eau de la partie méridionale de la villa. Cette nouvelle adduction s'inscrit en effet au sein d'aménagements importants intervenant lors de la seconde moitié du IVes. apr. J.-C., notamment la transformation en unité agricole du long bassin d'agrément B2 dans la zone I et la restructuration de l'espace 30 dans la zone IV.

Dans l'état actuel de nos connaissances, il est difficile de déterminer le tracé et la fonction exacts de l'aqueduc C.4. Néanmoins, la présence de cette canalisation, sans lien

121. Cette construction, associée à unc première phase de nivellement du secteur (LS 10064), est contemporaine du bâtiment E17. Elle correspond à quatre blocs non taillés visibles sur une à deux assises sur $1,15 \mathrm{~m}$ de long, sur lesquels s'appuic le troncon (4b et contre lesquels est venue saccoler la canalisation ( 66 .

122. Ia decouverte, dans le remblai de fondation de la strucure $(6$ (US 10056), d'un plat complet en ceramique grise tardive de fabrication locale (forme héritée des céramiques à pisolithes), daté de la seconde moitic du IV" s. apr. J.-C. et d'un nummus de Constance II de type Arles de la période $350-355$ (n" $10056-(01 / 01)$ précise le terminus ante quem de cette construction dans la seconde moitié du IV ${ }^{\circ}$ s. apr. J.-C: (ćtat Vb), lors de la restructuration de la pars urbana (zones I, II et IV). 
apparent avec l'alimentation en eau de la villa, présente un certain nombre de caractéristiques et révèle la complexité de l'aménagement de ce site.

Certaines caractéristiques liées à la technique de construction de cet ouvrage se retrouvent sur nombre d'aqueducs ruraux et urbains : mur de soutènement en opus vitattum, fond du specus de dimension moyenne en béton de tuileau et bourrelets d'étanchéité bordés de piédroits enduits de mortier de tuileau, profil en $U$ avec section rectangulaire, couverture avec dalles et tegulae à plat (Gateau et al., 1996, p. 156 et p. 239 ; Brun dir., 1999, p. 263 ; Lugand, Bermond, 2001, p. 256). En revanche, certaines interrogations demeurent en ce qui concerne la longueur totale de l'ouvrage, la pente moyenne générale, la fonction exacte du muret de bordement M52, le volume d'eau journalier transporté dans l'aqueduc $\mathrm{C} 4$, avant et après la construction de l'aqueduc C6 et l'impact de ce dernier sur son fonctionnement...

La prise d'eau de cet ouvrage reste également à déterminer, mais elle pourrait correspondre à une dérivation de l'aqueduc principal alimentant directement la villa depuis les sources présentes sur le contrefort oriental du Cengle, entre 700 et $1500 \mathrm{~m}$ au nord du site ( $336 \mathrm{~m}$ et $370 \mathrm{~m}$ d'altitude) (fig. 135).

$\mathrm{Au}$ vu de la présence du fond bétonné du specus, de la qualité de construction du mur de soutènement et son tracé parallèle à la zone d'habitat et au paléochenal 13010, cette canalisation ne peut être attribuée à un simple " égout ». Afin d'évacuer les eaux usées de la partie résidentielle de la villa, le conduit se serait déversé directement à l'est dans le ruisseau alors qu'il longe l'habitat et se poursuit bien au-delà du cours d'eau antique (voir supra, p. 15う̄-156). Sur la rive gauche de ce cours d'eau, le tracé de l'aqueduc pourrait bifurquer de nouveau, s'orienter au sud en longeant la rivière et être alors conservé sous le chemin d'accès à la propriété. Cette hypothèse se fonde sur les observations faites lors de sondages réalisés en 2002, sous la direction de C. Allinne et C. Miramont, sur la rive droite du ruisseau actuel de la Naïsse où ont été identifiés des dépôts sableux de berge interstratifiés à la base avec quelques niveaux de débordement. Des blocs épars, des fragments de mortier et des niveaux tassés montrent vraisemblablement un niveau de circulation sans qu'il soit possible de le dater.

$\mathrm{Si}$ les exemples d'aqueducs alimentant des villae sont fréquents en Gaule Narbonnaise (Gateau et al., 1996 ; Brun dir., 1999 ; Gateau, Gazenbeek, 1999 ; Lugand, Bermond, 2001), très peu en revanche témoignent d'une dérivation se dirigeant vers un bâtiment agricole annexe ou une installation hydrau- lique: villa de Seneymes à Martigues dans les Bouches-duRhône (Gateau et al., 1996, p. 239) et villa des Mesclans à La Crau dans le Var (Brun dir., 1999, p. 353-354).

L'inventaire cartographique de l'occupation du sol durant le Haut-Empire et l'Antiquité tardive a révélé autour de Richeaume, sur une superficie de 300 ha environ, un certain nombre de vestiges inhérents à la villa (fig. 135) : des établissements ruraux (Richeaume II, Prébois) et des installations agricoles sans doute plus modestes (Boasse, Richeaume IV, V, VI, VII et VIII) souvent hérités du second âge du Fer et, très récemment, plusieurs sépultures (incinérations du HautEmpire et inhumations tardives sous bâtière) ainsi qu'un enclos ou bâtiment funéraire révélant sans doute la présence d'une nécropole sur la rive droite de la Naïse (Richeaume XIII) ${ }^{123}$. Trois des établissements isolés sont localisés au sud (Richeaume VI et VII) et à l'est (Richeaume II) de la villa et de l'aqueduc C4. Le site de Richeaume II, implanté sur la rive gauche de la Naïsse, au sud des sépultures de Richeaume XIII, correspondrait à un vaste bâtiment agricole : petit canal d'adduction nord-sud taillé dans un bloc de safre et fragments de dolium et d'amphores recueillis en prospection. La nature des sites de Richeaume VI et le plus proche de la zone III, Richeaume VI, est plus difficile à cerner. La présence de l'aqueduc $\mathrm{C} 4$ sur ce versant oriental du domaine, dont la pente à forte déclivité a nécessité la construction de cette imposante substruction, pourrait être ainsi associée à l'alimentation en eau d'un de ces bâtiments voisins dont la nature reste à définir : moulin, citerne, huilerie, bassin... (Mocci, 1999 ; Walsh, Mocci, 2003).

Cette première analyse sur les aménagements hydrauliques antiques du versant oriental du domaine de Richeaume I et leur intégration au sein d'une dynamique de l'habitat pourrait alimenter le débat sur l'identification de ce type de site (Leveau, 2002). Correspond-il à un centre domanial majeur d'exploitation et de production entrainant un regroupement d'établissements ou bien au développement d'une agglomération de type vicus?

123. Ces vestiges ont été découverts en juillet 2004 lors de la réalisation d'une tranchée de canalisation au nord du site de Richeaume II ; une fouille de sauvetage a été entreprise du $1^{\text {cr }}$ au 7 août 2004 sous la direction de F. Mocci avec la collaboration de S. Ait-Ouméziane, V. Bignani, V. Dumas, F. Marty et B. Perez. Cne prospection géophysique du secteur a été menée en avril 2005 par l'université de York et le centre Camille-Jullian, sous la direction de F. Mocci et K. Walsh. 


\begin{abstract}
ANNEXE

\section{PREMIÈRES DONNÉES SUR LE PUITS} DE LA BASTIDE DE RICHEAUME

En liaison avec une éventuelle pérennité à l'époque médićvale, un puits en petit appareil (P1), intégré dans la rénovation de la bastide actuelle, pourrait être associé à l'aqueduc principal de la villa de Richeaume (fig. 136 et 146). Cet ouvrage, d'un diamètre intéricur de $1,08 \mathrm{~m}$ au sommet (cotes NGF : $319,48 \mathrm{~m}$ et $319,51 \mathrm{~m}$ ) et $1,06 \mathrm{~m}$ au fond (cotes NGF : $317,07 \mathrm{~m}$ à $317,22 \mathrm{~m}$ ), est conservé sur une hauteur de 2,44 à 2,64 m soit 22 à 24 assises de moellons plus ou moins équarris et liés au mortier de chaux. Des traces de dépôts carbonatés et

Cet ouvrage correspondrait au puits d'un moulin mentionné au plus tôt au $\mathrm{XV}^{\mathrm{c}}$ s. dans la "bastido des Richelmes " ${ }^{24}$. Son approvisionnement en eau proviendrait de l'aqueduc situć au nord, mais il aurait pu également y avoir un bassin de captage des eaux en amont ${ }^{125}$. Au fond du puits servant de colonne de pression, l'eau serait dirigée vers une roue hydraulique horizontale. Dans l'état actuel des recherches en cours avec la collaboration de H. Amouric, J.-L. Paillet et J.-M. Gassend ${ }^{126}$, aucun élément archéologique ne permet de préciser si la construction et l'utilisation de cet ouvrage remontent à l'époque antique: puits perdu, puits de moulin, turbine, etc.?
\end{abstract} d'enduits sont encore partiellement visibles sur les parois qui ne comportent, en revanche, aucune trace de béton de tuileau. De l'alimentation en eau ne subsiste qu'une assise de trois petits blocs équarris correspondant sans doute au piédroit nord d'une canalisation de direction ouest-est (cote: $319,58 \mathrm{~m}$ NGF). Des dalles posées à plat sur le substrat argileux constituent le fond du puits et du canal d'évacuation des eaux dont la pente de direction nord-ouest - sud-est n'est conservée que sur une longueur de $84 \mathrm{~cm}$. Les parements et la couverture du canal d'évacuation sont constitués de gros blocs de pierre rectangulaires plus ou moins équarris et liés au mortier de chaux. La largeur du conduit, décentré par rapport à l'axe vertical du puits, est de $54,50 \mathrm{~cm}$ au départ, $49 \mathrm{~cm}$ à $33 \mathrm{~cm}$ ct $47 \mathrm{~cm}$ à $40 \mathrm{~cm}$, sa hauteur est de $54 \mathrm{~cm}$.
124. Les données issues des archives cadastrales de Puyloubier, étudiées et transmises par H. Amouric, mentionnent en 1440 la présence de moulin au lieu-dit Aygas Vivas (.MF Arbaud 27, archives départementales des Bouches-du-Rhône) et en 1556 "lous mollhius des Richelmes " (fol. XXXIII et fol. I.XII, 151 E CC6, archives départementales des Bouches-du-Rhône).

125. Un bassin de captage, en grande partie enfoui, a été identifié récemment sur le tracé de la canalisation d'alimentation du domaine (environ $200 \mathrm{~m}$ en amont).

126. Nous tenons à remercier H. Amouric, J.-M. Gassend et J.-L. Paillet pour leur disponibilité ct leur généreuse contribution à cette étude.

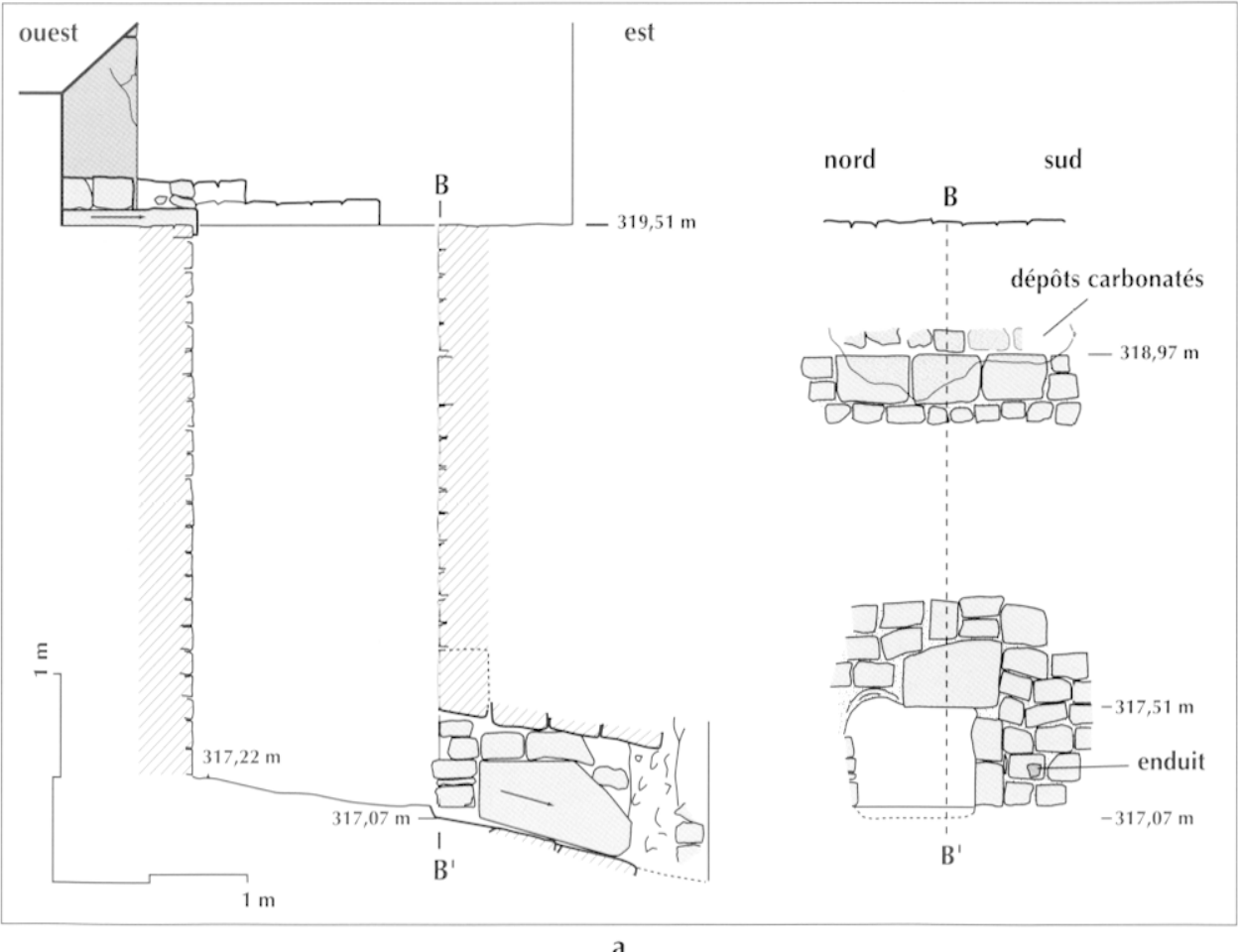

a

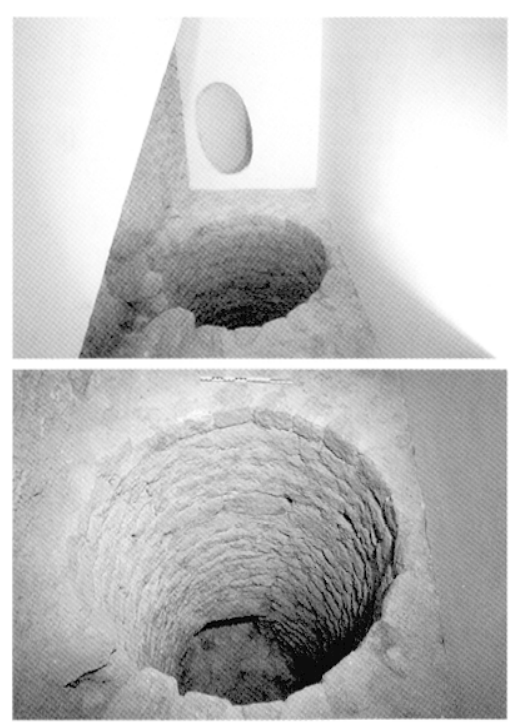

b

Fig. 146 - Coupe transversale et vues depuis l'ouest du puits Pl et du canal deracuation des eanex dans la bastide de Richeaume (D)AO V. Dumas et cliches (: Durand, (NRS). 